\title{
Modifications of Phenolic Compounds, Biogenic Amines, and Volatile Compounds in Cabernet Gernishct Wine through Malolactic Fermentation by Lactobacillus plantarum and Oenococcus oeni
}

\author{
Shao-Yang Wang ${ }^{1,2}{ }^{\oplus}$, Hai-Zhen Zhu ${ }^{3}$, Yi-Bin Lan ${ }^{4}$, Ruo-Jin Liu ${ }^{1}$, Ya-Ran Liu ${ }^{1}$, \\ Bo-Lin Zhang ${ }^{1}{ }^{(1)}$ and Bao-Qing Zhu ${ }^{1, *}$ \\ 1 Beijing Key Laboratory of Forestry Food Processing and Safety, Department of Food Sciences, \\ College of Biological Sciences and Biotechnology, Beijing Forestry University, Beijing 100083, China; \\ shaoyang.wang@uq.edu.au (S.-Y.W.); 13244000808@163.com (R.-J.L.); liuyaran@bjfu.edu.cn (Y.-R.L.); \\ zhangbolin888@163.com (B.-L.Z.) \\ 2 Centre for Nutrition and Food Sciences, Queensland Alliance for Agriculture and Food Innovation (QAAFI), \\ The University of Queensland, Brisbane 4108, Australia \\ 3 Institute of Architectural Civil Engineering, Langfang Normal University, Langfang, Hebei 065000, China; \\ 1331383@lfnu.edu.cn \\ 4 Cool Climate Oenology and Viticulture Institute (CCOVI), Brock University, St. Catharines, ON L2S 3A1, \\ Canada; vf_ylan@brocku.ca \\ * Correspondence: zhubaoqing@bjfu.edu.cn; Tel.: +86-106-2338-221
}

Received: 27 November 2019; Accepted: 15 January 2020; Published: 20 January 2020

\begin{abstract}
Malolactic fermentation is a vital red wine-making process to enhance the sensory quality. The objective of this study is to elucidate the starter cultures' role in modifying phenolic compounds, biogenic amines, and volatile compounds after red wine malolactic fermentation. We initiated the malolactic fermentation in Cabernet Gernishct wine by using two Oenococcus oeni and two Lactobacillus plantarum strains. Results showed that after malolactic fermentation, wines experienced a content decrease of total flavanols and total flavonols, accompanied by the accumulation of phenolic acids. The Lactobacillus plantarum strains, compared to Oenococcus oeni, exhibited a prevention against the accumulation of biogenic amines. The malolactic fermentation increased the total esters and modified the aromatic features compared to the unfermented wine. The Lactobacillus plantarum strains retained more aromas than the Oenococcus oeni strains did. Principal component analysis revealed that different strains could distinctly alter the wine characteristics being investigated in this study. These indicated that Lactobacillus plantarum could serve as a better alternative starter for conducting red wine malolactic fermentation.
\end{abstract}

Keywords: Lactobacillus plantarum; Oenococcus oeni; malolactic fermentation; phenolic compounds; biogenic amines; volatile compounds

\section{Introduction}

Malolactic fermentation is a vital red wine-making process after alcoholic fermentation since it can significantly improve the taste quality of red wine through converting the tart-taste malic acid into softer-taste lactic acid [1]. It also plays an important role in enhancing the aromatic complexity of wine via metabolism of nutrients by lactic acid bacteria [2-4]. It has been known that indigenous lactic acid bacteria naturally present in grapes must initiate spontaneous malolactic fermentation in wine $[5,6]$. This could trigger the production of undesirable metabolites (such as volatile acids and biogenic amines), which lowers the sensory quality of wine and further induces safety concerns $[7,8]$. 
Therefore, searching for good lactic acid bacteria strains for a reliable malolactic fermentation has been of particular interest in the wine industry [7]. Oenococcus oeni is a major group of lactic acid bacteria being widely applied as a commercial starter culture for malolactic fermentation due to its high tolerance to harsh wine environmental conditions (high acidity and high alcohol) [3,7,9]. Additionally, Lactobacillus plantarum has recently gained much attention in the wine industry as an alternative starter culture [3,7,9-14]. It has been reported that L. plantarum possesses the similar resistant capacity against the wine harsh environment as O. oeni does $[11,15,16]$. More importantly, L. plantarum was found to release a more diverse array of enzymes during malolactic fermentation, potentially improving the organoleptic features of wine [17-20].

Phenolic compounds play essential roles in determining wine appearance, bitterness, and astringency $[10,21]$. They are biosynthesized as the secondary metabolites in grapes and are directly extracted into wine during the maceration [22]. Phenolic compounds have been reported to affect the growth and metabolism of lactic acid bacteria during malolactic fermentation [23-27]. However, their compositional alteration caused by lactic acid bacteria during malolactic fermentation has not been well documented. In addition, volatile compounds have been confirmed to affect aromatic features of wine [28]. These grape-derived compounds determine the varietal aroma, and the fermentation and aging emphasize the fermentative and aging aromas of wine [29]. Most of the studies in the last decade were focused on the composition alteration of volatiles in wine under malolactic fermentation by using different strains of $O$. oeni [2,30-33]. Some recent studies have started to investigate the effect of $L$. plantarum as the single starter culture or a combined culture with $O$. oeni on the volatile alteration $[13,34,35]$. However, most these studies did not systematically investigate the change of wine overall aroma by neglecting odor activity values of volatile compounds. Besides phenolic and volatile compounds, biogenic amines could be yielded during malolactic fermentation and these small molecules could introduce safety concerns in wine. It was reported that the excessive intake of biogenic amines could cause headaches, blushing, itching, and skin irritation to humans, while the alcohol in wine even intensifies their toxicity through inhibiting the enzymes for biogenic amine catabolism [36]. The commercial starter culture, O. oeni, has been confirmed to produce biogenic amines during malolactic fermentation [37-41]. However, the producibility of biogenic amines via L. plantarum during malolactic fermentation has not been well studied [12,42].

In the present study, we selected four lactic acid bacteria strains, including two L. plantarum and two O oeni, to initiate the malolactic fermentation of Cabernet Gernischt wine. The composition of phenolic compounds, biogenic amines, and volatile compounds in these wine samples were analyzed and compared. The objective of this study was to elucidate the effect of different starter cultures on the modification of these organoleptic attributes in wine. The findings from this study could provide practical knowledge on quality control and improvement of red wine.

\section{Materials and Methods}

\subsection{Alcoholic Fermentation, Malolactic Fermentation, and Chemicals}

Details of alcoholic fermentation of Cabernet Gernischt wine can be found in our published work [43]. The initial red wine before malolactic fermentation had an alcohol level of $12.5 \%(v / v)$, pH 3.46, total acid $4.28 \mathrm{~g} / \mathrm{L}$, volatile acid $0.14 \mathrm{~g} / \mathrm{L}$. The initial concentration of malic acid and lactic acid were 2.51 and $0.50 \mathrm{~g} / \mathrm{L}$, respectively.

Four lactic acid bacteria strains were used in the present study, including two L. plantarum strains (Lp39, CICC6240 and C8-1, CICC23138) and two O. oeni strains (Viniflora ${ }^{\circledR \circledR}$ Oenos and CiNe). The Lp39 and C8-1 strains were received from China Centre of Industrial Culture Collection (Beijing, China). The Viniflora ${ }^{\circledR}$ Oenos and CiNe strains were the commercial starter cultures purchased from Chr. Hansen (Hoersholmm, Denmark). Details of culture preparation, inoculation, malolactic fermentation procedure, and physicochemical data can be found in our published work [43]. 
Methanol, acetonitrile, and formic acid were HPLC grade and purchased from Fisher (Fairlawn, NJ, USA). Sodium azide was purchased from Sangon (Shanghai, China). Diethyl ethoxymethylenemalonate (derivatization reagent) and external standards of phenolic compounds, biogenic amines, and volatile compounds were all purchased from Sigma-Aldrich (St. Louis, MO, USA). 4-Methyl-2-pentanol (internal standard for volatiles extraction) was also a product from Sigma-Aldrich (St. Louis, MO, USA).

\subsection{Analysis of Phenolic Compounds}

An Agilent 1200 Series high-performance liquid chromatography coupled with an Agilent 6410 triple quadrupole mass spectrometer (HPLC-QqQ-MS/MS, Agilent Technologies, Santa Clara, CA, USA) was used to analyze phenolic compounds in the wine samples according to a published method [21]. The wine sample $(1.0 \mathrm{~mL})$ was filtered through a $0.45-\mu \mathrm{m}$ polyether sulphone membrane, and then the filtered wine sample $(1.0 \mu \mathrm{L})$ was directly injected to HPLC. Phenolic compounds in the wine samples were separated on a Poroshell 120 EC-C18 column $(150 \times 2.1 \mathrm{~mm}, 2.7 \mu \mathrm{m}$, Agilent Technologies, Santa Clara, CA, USA) with a flow rate at $0.4 \mathrm{~mL} / \mathrm{min}$. Mobile phase consisted of (A) $0.1 \% v / v$ formic acid in water and (B) $0.1 \% v / v$ formic acid in acetonitrile:methanol $(1: 1, v / v)$. The gradient elution program was as follows: $0-28 \mathrm{~min}, 10 \% \mathrm{~B}$ to $46 \% \mathrm{~B} ; 28-29 \mathrm{~min}, 46 \% \mathrm{~B}$ to $10 \% \mathrm{~B}$; and $29-34 \mathrm{~min}, 10 \% \mathrm{~B}$ isocratic. The column was maintained at $55{ }^{\circ} \mathrm{C}$ during the gradient elution program. A negative electrospray ionization was used on QqQ-MS/MS with a spray voltage at $4 \mathrm{kV}$, gas temperature at $350{ }^{\circ} \mathrm{C}$, and nebulizer pressure at 35 psi. The ion source temperature was set at $150{ }^{\circ} \mathrm{C}$. Multiple reaction monitoring mode for the transition of the precursor to product ion was used to identify phenolic compounds in the wine samples. The external standard (procyanidin B1, procyanidin B2, procyanidin $\mathrm{C} 1,(+)$-catechin, (-)-epicatechin, (-)-epigallocatechin and gallocatechin, myricetin, myricetin-3-O-glucoside, quercetin-3-O-galactoside, quercetin-3-O-glucuronide, dihydroquercetin, syringetin-3-O-glucoside, gallic acid, protocatechuic acid, caffeic acid, and 4-hydroxycinnamic acid) were used to quantify flavanols, flavonols, and phenolic acids, respectively. Each wine sample was analyzed in duplicate.

\subsection{Analysis of Biogenic Amines}

A published derivatization-liquid chromatography method was used to analyze biogenic amines in these wine samples with minor modifications [44]. In brief, the wine sample (500 $\mu \mathrm{L})$ was mixed with $10 \mu \mathrm{L}$ of $1 \mathrm{~g} / \mathrm{L}$ L-2-aminoadipic acid (internal standard), $375 \mu \mathrm{L}$ of methanol, $15 \mu \mathrm{L}$ of diethyl ethoxymethylenemalonate, and $875 \mu \mathrm{L}$ of $1 \mathrm{~mol} / \mathrm{L}$ borate buffer ( $\mathrm{pH}$ 9.0). The mixture was sonicated for $30 \mathrm{~min}$ and then heated at $70^{\circ} \mathrm{C}$ in a water bath for $2 \mathrm{~h}$. After the mixture was cooled down to the room temperature, the mixture was filtered through $0.22 \mu \mathrm{m}$ nylon filters and then $20 \mu \mathrm{L}$ filtered wine sample was injected to HPLC. A Shimadzu LC-20AT LC system (Shimadzu, Kyoto, Japan) with a Venusil XSB C18 column $(4.6 \times 250 \mathrm{~mm}, 5 \mu \mathrm{m}$, Bonna-Agela Technologies Co. Ltd., Tianjin, China) was used to analyze the biogenic amine derivatives. The mobile phase was comprised of (A) acetonitrile:methanol $(4: 1, v / v)$ and (B) $25 \mathrm{mM}$ acetate buffer ( $\mathrm{pH} 5.8)$ containing $0.02 \%$ sodium azide. The flow rate was set at $0.9 \mathrm{~mL} / \mathrm{min}$ with a column temperature maintained at $16{ }^{\circ} \mathrm{C}$ during the elution program. The elution was programed as follows: $0-20 \mathrm{~min}, 90 \% \mathrm{~B}$ isocratic; $20-30.5 \mathrm{~min}, 90 \% \mathrm{~B}$ to $83 \% \mathrm{~B} ; 30.5-33.5 \mathrm{~min}, 83 \% \mathrm{~B}$ isocratic; $33.5-65 \mathrm{~min}, 83 \% \mathrm{~B}$ to $73 \% \mathrm{~B}$; $65-73 \mathrm{~min}, 73 \% \mathrm{~B}$ to $28 \% \mathrm{~B} ; 73-78 \mathrm{~min}, 28 \% \mathrm{~B}$ to $18 \% \mathrm{~B}$; $78-82 \mathrm{~min}$, $28 \% \mathrm{~B}$ to $0 \% \mathrm{~B}$; $82-85 \mathrm{~min}, 0 \% \mathrm{~B}$ isocratic; $85-90 \mathrm{~min}, 0 \% \mathrm{~B}$ to $90 \% \mathrm{~B}$; and $90-93 \mathrm{~min}, 90 \% \mathrm{~B}$ isocratic. A model wine matrix, consisting of $14 \% \mathrm{v} / \mathrm{v}$ ethanol and $5 \mathrm{~g} / \mathrm{L}$ of tartaric acid in water ( $\mathrm{pH}$ adjusted to 3.8 using $5 \mathrm{M} \mathrm{NaOH}$ ), was mixed with the external biogenic amine standards. The standards wine matrix was analyzed under the same derivatization-liquid chromatography method. The quantitation was performed using the regression curves generated through the peak ratio of external over internal standards versus the concentrations of the external standards. Each sample was analyzed in duplicate. 


\subsection{Analysis of Volatile Compounds}

Volatile compounds in these wine samples were extracted using headspace solid-phase microextraction and then analyzed using gas chromatography-mass spectrometry [45]. Briefly, the wine sample $(5.0 \mathrm{~mL})$ was mixed with $1 \mathrm{~g} \mathrm{NaCl}, 10 \mu \mathrm{L}$ of $1.0018 \mathrm{~g} / \mathrm{L}$ 4-methyl-2-pentanol (internal standard) in a 15-mL glass vial containing a magnetic stirrer. The vial was capped tightly with a PTFE-silicon septum. The mixture was equilibrated at $40^{\circ} \mathrm{C}$ for $30 \mathrm{~min}$ under an agitation at $300 \mathrm{rpm}$. Afterwards, a 50/30 $\mu$ m divinylbenzene/carboxen/polydimethylsiloxane fiber (DVB/CAR/PDMS; Supelco, Bellefonte, PA, USA) was inserted to the headspace of the vial for $30 \mathrm{~min}$ to adsorb volatiles at $40^{\circ} \mathrm{C}$ under agitation at $300 \mathrm{rpm}$. Afterwards, the fiber was removed from the headspace of the vial and immediately inserted to the GC injector to release the volatiles at $250^{\circ} \mathrm{C}$ for $8 \mathrm{~min}$. An Agilent 6890 gas chromatography coupled with an Agilent 5975 mass spectrometry (Agilent Technologies, Santa Clara, CA, USA) was used to analyze the volatile compounds in the wine samples. An HP-Innowax capillary column (60 $\mathrm{m} \times 0.25 \mathrm{~mm} \times 0.25 \mu \mathrm{m}, \mathrm{J} \& W$ Scientific, San Francisco, CA, USA) was used to separate the volatile compounds under a carrier gas (helium) flow rate at $1 \mathrm{~mL} / \mathrm{min}$. The oven temperature program was as follows: held at $50{ }^{\circ} \mathrm{C}$ for $1 \mathrm{~min}$, increased to $220{ }^{\circ} \mathrm{C}$ at a $3{ }^{\circ} \mathrm{C} / \mathrm{min}$ rate, and held at $220{ }^{\circ} \mathrm{C}$ for $5 \mathrm{~min}$. The temperature set at the interface and the ion source on the GC-MS were 280 and $230{ }^{\circ} \mathrm{C}$, respectively. All mass scan from $\mathrm{m} / \mathrm{z} 30$ to $\mathrm{m} / \mathrm{z} 350$ under a selective ion mode was conducted with the electron impact set at $70 \mathrm{eV}$. A C6 to C24 n-alkane series (Supelco, Bellefonte, PA, USA) was analyzed using the same chromatography condition to calculate the retention indices. Volatile compounds with their reference standard available were identified by comparing their mass spectrum and retention indices with their corresponding standard. Volatile compounds without the available standard were tentatively identified through comparing their retention indices and mass spectrum with the NIST11 library and the retention indices of references in our local library. Regarding the quantitation of volatile compounds, the external volatile standards were dissolved in ethanol and then diluted to 15 successive levels in the wine model matrix containing $14 \%$ v/ $/$ ethanol and $5 \mathrm{~g} / \mathrm{L}$ of tartaric acid in water $(\mathrm{pH}$ adjusted to 3.8 using $5 \mathrm{M} \mathrm{NaOH}$ ). The extraction and analysis of these standard samples followed the same volatiles analysis procedure as the wine samples. Standard curve of each volatile was established through the peak ratio of target volatile over internal standard versus the concentration of target volatile. For volatile compounds with the reference standard, the quantitation was achieved using their corresponding reference standard. Volatiles without the available standard were quantified using the standard that exhibited the same functional group and similar $C$ atom numbers as the volatile. Each wine sample was analyzed in duplicate.

\subsection{Odor Activity Value and Aroma Profile}

Odor activity value $(\mathrm{OAV})$ is a critical indicator to reflect the aromatic contribution of a volatile. The OAV is determined by the volatile concentration over its perception threshold reported in the literature. A volatile compound with OAV greater than 1 (esters with OAV above 0.1) significantly contributes its aroma features. Aroma profile in wine is divided into seven series, including fruity, floral, herbaceous, caramel, balsamic, chemical, and fatty aromas. The overall intensity of each aroma series was calculated through summing up the OAV of the volatiles that have significant aromatic contributions.

\subsection{Statistical Analysis}

Data were expressed as the mean \pm standard deviation. One-way analysis of variance (ANOVA) was carried out to compare the means under Tukey's honestly significant difference at a significant level of 0.05 using SPSS Statistics 23 (IBM, New York, NY, USA). Principal component analysis was conducted using XLSTAT (Addinsoft, New York, NY, USA). Only the variables with a significant difference between wine samples were included. 


\section{Results and Discussion}

\subsection{Phenolic Compounds}

A total of 17 phenolic compounds including seven flavanols, six flavonols, and four phenolic acids were found in these wine samples (Table 1). The total phenolic compounds before the malolactic fermentation was $95.72 \mathrm{mg} / \mathrm{L}$. Although the total concentration of phenolic compounds was not altered by any strain, a significant decrease in total flavanols was found, where the sample with C8-1 displayed the lowest. A slight reduction in total flavonols and similar content of total flavonols were also observed after the malolactic fermentation. It is worth noting the dramatic increase of gallic acid and protocatechuic acid in the sample with Lp39. The caffeic acid and 4-hydroxycinnamic acid were not quantified before the malolactic fermentation. However, a significant accumulation of these two phenolic acids was observed in three fermented samples except C8-1. The caffeic acid and 4-hydroxycinnamic acid in wines with $O$. oeni had doubled concentrations compared to samples with Lp39.

Table 1. Phenolic compounds in Cabernet Gernischt wine before and after malolactic fermentation using Lactobacillus plantarum (C8-1 and Lp39) and Oenococcus oeni (Oenos and CiNe).

\begin{tabular}{|c|c|c|c|c|c|}
\hline Phenolic Compounds ${ }^{1}$ & $\begin{array}{c}\text { Before Malolactic } \\
\text { Fermentation }\end{array}$ & $\begin{array}{l}\text { L. plantarum } \\
\text { C8-1 }\end{array}$ & $\begin{array}{l}\text { L. plantarum } \\
\text { Lp39 }\end{array}$ & $\begin{array}{l}\text { O. oeni } \\
\text { Oenos }\end{array}$ & $\begin{array}{l}\text { O. oеni } \\
\text { CiNe }\end{array}$ \\
\hline Procyanin B1 & $17.28 \pm 0.33 \mathrm{~B}$ & $16.64 \pm 0.39 \mathrm{AB}$ & $15.69 \pm 0.70 \mathrm{~A}$ & $16.53 \pm 0.52 \mathrm{AB}$ & $15.80 \pm 0.37 \mathrm{~A}$ \\
\hline Procyanin B2 & $4.72 \pm 0.06 \mathrm{~B}$ & $5.09 \pm 0.10 \mathrm{C}$ & $4.49 \pm 0.22 \mathrm{AB}$ & $4.20 \pm 0.15 \mathrm{~A}$ & $4.34 \pm 0.08 \mathrm{~A}$ \\
\hline Procyanin C1 & $2.74 \pm 0.09 \mathrm{~B}$ & $2.77 \pm 0.41 \mathrm{~B}$ & $2.02 \pm 0.05 \mathrm{~A}$ & $2.67 \pm 0.18 \mathrm{AB}$ & $2.91 \pm 0.34 \mathrm{~B}$ \\
\hline Catechin & $20.71 \pm 0.46 \mathrm{~B}$ & $18.20 \pm 0.03 \mathrm{~A}$ & $16.71 \pm 0.85 \mathrm{~A}$ & $18.91 \pm 1.74 \mathrm{AB}$ & $18.20 \pm 0.18 \mathrm{~A}$ \\
\hline Epicatechin & $34.92 \pm 0.09 \mathrm{C}$ & $27.71 \pm 0.14 \mathrm{AB}$ & $25.13 \pm 1.03 \mathrm{~A}$ & $28.44 \pm 1.93 \mathrm{~B}$ & $27.94 \pm 0.03 \mathrm{~B}$ \\
\hline Epigallocatechin & $0.52 \pm 0.01 \mathrm{~B}$ & $0.47 \pm 0.02 \mathrm{~A}$ & $0.44 \pm 0.02 \mathrm{~A}$ & $0.56 \pm 0.01 \mathrm{~B}$ & $0.45 \pm 0.01 \mathrm{~A}$ \\
\hline Gallocatechin & $8.80 \pm 0.09 \mathrm{~A}$ & $8.87 \pm 0.20 \mathrm{~A}$ & $7.98 \pm 0.66 \mathrm{~A}$ & $9.13 \pm 0.68 \mathrm{~A}$ & $8.77 \pm 0.16 \mathrm{~A}$ \\
\hline Total flavanols & $89.70 \pm 0.21 \mathrm{C}$ & $79.75 \pm 0.19 \mathrm{AB}$ & $72.47 \pm 3.53 \mathrm{~A}$ & $80.43 \pm 5.18 \mathrm{~B}$ & $78.41 \pm 1.16 \mathrm{AB}$ \\
\hline Myricetin & $0.32 \pm 0.01 \mathrm{~B}$ & $0.23 \pm 0.01 \mathrm{~A}$ & $0.25 \pm 0.02 \mathrm{~A}$ & $0.26 \pm 0.02 \mathrm{~A}$ & $0.25 \pm 0.00 \mathrm{~A}$ \\
\hline Myricetin-glucoside & $2.78 \pm 0.03 \mathrm{C}$ & $2.41 \pm 0.03 \mathrm{AB}$ & $2.29 \pm 0.11 \mathrm{~A}$ & $2.52 \pm 0.09 \mathrm{~B}$ & $2.40 \pm 0.03 \mathrm{AB}$ \\
\hline Quercetin-galactoside & $0.50 \pm 0.00 \mathrm{~B}$ & $0.44 \pm 0.01 \mathrm{~A}$ & $0.42 \pm 0.02 \mathrm{~A}$ & $0.43 \pm 0.00 \mathrm{~A}$ & $0.44 \pm 0.02 \mathrm{~A}$ \\
\hline Quercetin-glucuronide & $0.42 \pm 0.03 \mathrm{~B}$ & $0.27 \pm 0.01 \mathrm{AB}$ & $0.16 \pm 0.07 \mathrm{~A}$ & $0.23 \pm 0.10 \mathrm{~A}$ & $0.24 \pm 0.02 \mathrm{~A}$ \\
\hline Dihydroquercetin & $0.12 \pm 0.00 \mathrm{AB}$ & $0.06 \pm 0.04 \mathrm{~A}$ & $0.06 \pm 0.02 \mathrm{AB}$ & $0.12 \pm 0.03 \mathrm{~B}$ & $0.08 \pm 0.00 \mathrm{AB}$ \\
\hline Syringetin-glucoside & $0.94 \pm 0.01 \mathrm{C}$ & $0.86 \pm 0.00 \mathrm{~B}$ & $0.81 \pm 0.01 \mathrm{~A}$ & $0.80 \pm 0.03 \mathrm{~A}$ & $0.80 \pm 0.00 \mathrm{~A}$ \\
\hline Total flavonols & $5.08 \pm 0.06 \mathrm{~B}$ & $4.27 \pm 0.09 \mathrm{~A}$ & $4.00 \pm 0.23 \mathrm{~A}$ & $4.35 \pm 0.27 \mathrm{~A}$ & $4.21 \pm 0.08 \mathrm{~A}$ \\
\hline Gallic acid & $0.68 \pm 0.42 \mathrm{~A}$ & $3.85 \pm 0.17 \mathrm{~B}$ & $6.65 \pm 0.17 \mathrm{D}$ & $6.17 \pm 0.03 \mathrm{D}$ & $5.31 \pm 0.40 \mathrm{C}$ \\
\hline Protocatechuic acid & $0.26 \pm 0.02 \mathrm{~B}$ & $0.08 \pm 0.01 \mathrm{~A}$ & $0.42 \pm 0.03 \mathrm{E}$ & $0.38 \pm 0.01 \mathrm{D}$ & $0.33 \pm 0.01 \mathrm{C}$ \\
\hline Caffeic acid & $\operatorname{tr}$ & $\operatorname{tr}$ & $4.08 \pm 0.41 \mathrm{~A}$ & $8.97 \pm 0.40 \mathrm{C}$ & $7.56 \pm 0.26 \mathrm{~B}$ \\
\hline 4-Hydroxycinnamic acid & $\operatorname{tr}$ & $\operatorname{tr}$ & $0.59 \pm 0.04 \mathrm{~A}$ & $1.13 \pm 0.09 \mathrm{~B}$ & $1.05 \pm 0.01 \mathrm{~B}$ \\
\hline Total phenolic acids & $0.94 \pm 0.41 \mathrm{~A}$ & $3.93 \pm 0.17 \mathrm{~B}$ & $11.74 \pm 0.65 \mathrm{C}$ & $16.64 \pm 0.46 \mathrm{E}$ & $14.24 \pm 0.68 \mathrm{D}$ \\
\hline Total phenolic compounds & $95.72 \pm 0.68 \mathrm{AB}$ & $87.95 \pm 0.11 \mathrm{~A}$ & $88.20 \pm 4.41 \mathrm{~A}$ & $101.42 \pm 5.92 \mathrm{~B}$ & $96.86 \pm 0.40 \mathrm{AB}$ \\
\hline
\end{tabular}

It has been confirmed that lactic acid bacteria could release numerous enzymes, such as gallate decarboxylase, $p$-coumaric acid decarboxylase, benzyl alcohol dehydrogenase, oxidoreductase, decarboxylase, and demethylases, in wine during malolactic fermentation. These enzymes could metabolize phenolic compounds, resulting in the reduction of phenolic compounds in wine after malolactic fermentation [27,46-49]. This could explain why phenolic compounds, especially flavanols and flavonols, exhibited a concentration decrease in the Cabernet Gernischt wines after the malolactic fermentation in the present study. It should be noted that lactic acid bacteria also have capacity of releasing tannases, and these enzymes could cleave the ester bonds in hydrolysable tannins to yield their corresponding phenolic acid monomers [50,51]. This could increase the accumulation of phenolic acids in wine, such as caffeic acid and 4-hydroxycinnamic acid in the present study. 


\subsection{Biogenic Amines}

The Cabernet Gernischt wine before the malolactic fermentation contained total biogenic amines at $7.29 \mathrm{mg} / \mathrm{L}$ (Table 2). Except C8-1, all other wine samples exhibited a significant increase in the total biogenic amines. Regarding the individual biogenic amine, histamine was the dominant one before or after the malolactic fermentation. Its accumulation was observed in three samples expect C8-1. Spermidine and putrescine also existed at a moderate amount. The O. oeni strains slightly reduced the content of putrescine after the malolactic fermentation, whereas the spermidine remained unchanged.

Table 2. Biogenic amines in Cabernet Gernischt wine before and after malolactic fermentation using L. plantarum (C8-1 and Lp39) and O. oeni (Oenos and CiNe).

\begin{tabular}{cccccc}
\hline Biogenic Amines ${ }^{1}$ & $\begin{array}{c}\text { Before Malolactic } \\
\text { Fermentation }\end{array}$ & $\begin{array}{c}\text { L. plantarum } \\
\text { C8-1 }\end{array}$ & $\begin{array}{c}\text { L. plantarum } \\
\text { Lp39 }\end{array}$ & $\begin{array}{c}\text { O. oeni } \\
\text { Oenos }\end{array}$ & $\begin{array}{c}\text { O. oeni } \\
\text { CiNe }\end{array}$ \\
\hline Histamine & $5.06 \pm 0.03 \mathrm{~A}$ & $4.87 \pm 0.16 \mathrm{~A}$ & $6.26 \pm 0.17 \mathrm{~B}$ & $8.20 \pm 0.28 \mathrm{C}$ & $8.04 \pm 0.35 \mathrm{C}$ \\
Spermine & $0.05 \pm 0.00 \mathrm{~A}$ & $0.07 \pm 0.00 \mathrm{~B}$ & $0.07 \pm 0.01 \mathrm{~B}$ & $0.07 \pm 0.00 \mathrm{~B}$ & $0.07 \pm 0.00 \mathrm{~B}$ \\
Spermidine & $0.89 \pm 0.10 \mathrm{AB}$ & $0.80 \pm 0.05 \mathrm{~A}$ & $0.96 \pm 0.01 \mathrm{~B}$ & $0.85 \pm 0.00 \mathrm{AB}$ & $0.93 \pm 0.03 \mathrm{AB}$ \\
Tyramine & $0.05 \pm 0.00 \mathrm{~B}$ & $0.02 \pm 0.01 \mathrm{~A}$ & $0.05 \pm 0.00 \mathrm{~B}$ & $0.02 \pm 0.01 \mathrm{~A}$ & $0.02 \pm 0.01 \mathrm{~A}$ \\
Putrescine & $1.10 \pm 0.02 \mathrm{C}$ & $1.05 \pm 0.02 \mathrm{BC}$ & $1.07 \pm 0.02 \mathrm{C}$ & $0.99 \pm 0.01 \mathrm{~B}$ & $0.91 \pm 0.05 \mathrm{~A}$ \\
Cadaverine & $0.02 \pm 0.00 \mathrm{C}$ & $0.01 \pm 0.00 \mathrm{~A}$ & $0.01 \pm 0.00 \mathrm{AB}$ & $0.01 \pm 0.00 \mathrm{BC}$ & $0.01 \pm 0.00 \mathrm{~A}$ \\
Phenethylamine & $0.13 \pm 0.03 \mathrm{~A}$ & $0.12 \pm 0.00 \mathrm{~A}$ & $0.11 \pm 0.02 \mathrm{~A}$ & $0.12 \pm 0.00 \mathrm{~A}$ & $0.11 \pm 0.00 \mathrm{~A}$ \\
Total biogenic amines & $7.29 \pm 0.11 \mathrm{~A}$ & $6.94 \pm 0.21 \mathrm{~A}$ & $8.54 \pm 0.23 \mathrm{~B}$ & $10.28 \pm 0.28 \mathrm{C}$ & $10.08 \pm 0.33 \mathrm{C}$ \\
\hline 1 Data are expressed as mean \pm standard deviation of duplicate tests. Different letters in the same row indicate \\
significant difference at $p<0.05$.
\end{tabular}

Biogenic amines negatively influence the quality of wine since these metabolites could potentially bring safety concerns to consumers [36]. In wine, the amino acids are the major precursors of biogenic amines and these molecules could be metabolized by lactic acid bacteria thus to yield biogenic amines [52]. Therefore, lactic acid bacteria strains have been investigated and screened as starter cultures in the wine industry in terms of their capacity of producing biogenic amines [14]. Different lactic acid bacteria species possess different abilities of metabolizing amino acids to yield biogenic amines, and the $O$. oeni strains have been reported with high production of biogenic amines [37,38]. For example, the malolactic fermentation in Falanghina wine by O. oeni doubled the histamine compared to that by L. plantarum [39]. Our results were consistent with this report. Meanwhile, L. plantarum strains have been reported to lack some enzymes as $O$. oeni, which convert amino acids into biogenic amines $[9,11,12,40,41]$. This might explain why the C8-1 fermented Cabernet Gernischt wine did not increase biogenic amines after malolactic fermentation in the present study.

\subsection{Volatile Compounds}

A total of 71 volatile compounds were detected in these Cabernet Gernischt wine samples (Table 3), including 24 esters, 14 higher alcohols, six aldehydes/ketones, seven fatty acids, 10 terpene derivatives, and 10 other volatiles. The most abundant groups were found to be higher alcohols, followed by esters, aldehydes/ketones, and then fatty acids. Terpene derivatives and other volatiles appeared to exist in these wine samples at a low-level range. 
Table 3. Volatile compounds in Cabernet Gernischt wine before and after malolactic fermentation using L. plantarum (C8-1 and Lp39) and O. oeni (Oenos and CiNe).

\begin{tabular}{|c|c|c|c|c|c|c|c|}
\hline Code & & $\mathrm{RI}^{1,2,3}$ & $\begin{array}{l}\text { Before Malolactic } \\
\text { Fermentation }\end{array}$ & $\begin{array}{l}\text { L. plantarum } \\
\text { C8-1 }\end{array}$ & $\begin{array}{l}\text { L. plantarum } \\
\text { Lp39 }\end{array}$ & $\begin{array}{l}\text { O. oeni } \\
\text { Oenos }\end{array}$ & $\begin{array}{l}\text { O. oeni } \\
\text { CiNe }\end{array}$ \\
\hline & $\begin{array}{c}\text { Esters } \\
\text { Acetate esters }\end{array}$ & & & & & & \\
\hline E1 & Ethyl acetate ${ }^{a}(\mathrm{mg} / \mathrm{L})$ & 801.9 & $41.31 \pm 0.15 B$ & $33.66 \pm 0.39 \mathrm{~A}$ & $39.44 \pm 1.24 \mathrm{~B}$ & $31.82 \pm 0.87 \mathrm{~A}$ & $31.09 \pm 1.23 \mathrm{~A}$ \\
\hline E2 & Isoamyl acetate ${ }^{a}$ & 1120.3 & $801.25 \pm 3.71 \mathrm{C}$ & $339.49 \pm 38.62$ B & $359.04 \pm 14.14 \mathrm{~B}$ & $287.38 \pm 10.61 \mathrm{AB}$ & $201.10 \pm 36.87 \mathrm{~A}$ \\
\hline \multirow[t]{2}{*}{ E3 } & Phenethyl acetate a & 1802.9 & $2.70 \pm 0.09 \mathrm{~B}$ & $7.14 \pm 0.23 \mathrm{C}$ & $2.25 \pm 0.04 \mathrm{~A}$ & $2.24 \pm 0.03 \mathrm{~A}$ & $2.22 \pm 0.03 \mathrm{~A}$ \\
\hline & $\begin{array}{c}\text { Total acetate esters }(\mathrm{mg} / \mathrm{L}) \\
\text { Ethyl esters }\end{array}$ & & $42.12 \pm 0.15 \mathrm{~B}$ & $34.01 \pm 0.43 \mathrm{~A}$ & $39.80 \pm 1.25 \mathrm{~B}$ & $32.11 \pm 0.88 \mathrm{~A}$ & $31.30 \pm 1.27 \mathrm{~A}$ \\
\hline E4 & Ethyl butanoate a & 1019.5 & $646.55 \pm 28.58 \mathrm{C}$ & $303.41 \pm 8.72 \mathrm{AB}$ & $364.32 \pm 25.93 \mathrm{~B}$ & $242.74 \pm 39.73 \mathrm{~A}$ & $217.36 \pm 22.17 \mathrm{~A}$ \\
\hline E5 & Ethyl hexanoate ${ }^{a}$ & 1229.2 & $543.51 \pm 0.41 \mathrm{C}$ & $263.05 \pm 37.13 \mathrm{AB}$ & $343.08 \pm 16.92 \mathrm{~B}$ & $255.41 \pm 12.59 \mathrm{AB}$ & $208.76 \pm 60.22 \mathrm{~A}$ \\
\hline E6 & Ethyl heptanoate a & 1329 & $1.42 \pm 0.01 \mathrm{~B}$ & $1.04 \pm 0.21 \mathrm{AB}$ & $1.12 \pm 0.09 \mathrm{AB}$ & $1.19 \pm 0.07 \mathrm{AB}$ & $0.64 \pm 0.32 \mathrm{~A}$ \\
\hline E7 & Ethyl lactate $^{a}(\mathrm{mg} / \mathrm{L})$ & 1336.2 & $4.96 \pm 0.20 \mathrm{~A}$ & $116.95 \pm 3.30 \mathrm{C}$ & $71.12 \pm 3.39 \mathrm{~B}$ & $66.22 \pm 5.32 \mathrm{~B}$ & $64.70 \pm 2.36 \mathrm{~B}$ \\
\hline E8 & $\begin{array}{c}\text { 2-Hydroxyisovaleric acid ethyl } \\
\text { ester }^{\mathrm{b}}(\mathrm{mg} / \mathrm{L})\end{array}$ & 1420 & $\operatorname{tr}$ & $2.80 \pm 0.05$ & $\operatorname{tr}$ & $\operatorname{tr}$ & $\operatorname{tr}$ \\
\hline E9 & Ethyl octanoate a & 1429.9 & $33.21 \pm 0.20 \mathrm{C}$ & $31.05 \pm 0.99 \mathrm{BC}$ & $32.94 \pm 0.34 \mathrm{C}$ & $27.17 \pm 0.02 \mathrm{~B}$ & $21.37 \pm 2.55 \mathrm{~A}$ \\
\hline E10 & Ethyl nonanoate a & 1531.3 & $0.78 \pm 0.00 \mathrm{~A}$ & $1.24 \pm 0.06 \mathrm{BC}$ & $1.18 \pm 0.04 \mathrm{ABC}$ & $1.44 \pm 0.17 \mathrm{C}$ & $0.80 \pm 0.16 \mathrm{AB}$ \\
\hline E11 & Ethyl decanoate ${ }^{b}$ & 1630.7 & $36.48 \pm 0.46 \mathrm{~B}$ & $34.26 \pm 0.08 \mathrm{~B}$ & $35.71 \pm 0.30 \mathrm{~B}$ & $34.36 \pm 0.88 \mathrm{~B}$ & $30.32 \pm 1.41 \mathrm{~A}$ \\
\hline E12 & Ethyl benzoate $\mathrm{b}$ & 1663.1 & $11.81 \pm 1.73 \mathrm{C}$ & $6.51 \pm 0.30 \mathrm{~A}$ & $8.08 \pm 0.11 \mathrm{AB}$ & $8.57 \pm 0.21 \mathrm{ABC}$ & $11.15 \pm 0.47 \mathrm{BC}$ \\
\hline E13 & Diethyl succinate a & 1667.5 & $124.19 \pm 15.40 \mathrm{~A}$ & $263.66 \pm 14.01 \mathrm{~B}$ & $226.67 \pm 0.77 \mathrm{~B}$ & $137.73 \pm 9.62 \mathrm{~A}$ & $130.25 \pm 9.63 \mathrm{~A}$ \\
\hline E14 & Ethyl dodecanoate ${ }^{a}$ & 1826.7 & $29.45 \pm 0.18 \mathrm{~A}$ & $29.21 \pm 0.08 \mathrm{~A}$ & $29.52 \pm 0.14 \mathrm{~A}$ & $29.85 \pm 0.82 \mathrm{~A}$ & $29.19 \pm 0.16 \mathrm{~A}$ \\
\hline E15 & Ethyl dihydrocinnamate ${ }^{b}$ & 1867 & $2.25 \pm 0.09 \mathrm{~B}$ & $1.66 \pm 0.10 \mathrm{~A}$ & $1.76 \pm 0.12 \mathrm{~A}$ & $1.75 \pm 0.01 \mathrm{~A}$ & $1.54 \pm 0.06 \mathrm{~A}$ \\
\hline E16 & Ethyl isopentyl succinate ${ }^{b}$ & 1878 & $14.95 \pm 1.89 \mathrm{~B}$ & $16.39 \pm 1.98 \mathrm{~B}$ & $15.27 \pm 2.00 \mathrm{~B}$ & $5.47 \pm 1.88 \mathrm{~A}$ & $4.39 \pm 1.73 \mathrm{~A}$ \\
\hline E17 & Ethyl cinnamate ${ }^{b}$ & 2079.3 & $54.32 \pm 14.90 \mathrm{~B}$ & $29.63 \pm 2.42 \mathrm{AB}$ & $25.12 \pm 0.70 \mathrm{~A}$ & $22.56 \pm 1.17 \mathrm{~A}$ & $20.70 \pm 0.31 \mathrm{~A}$ \\
\hline \multirow[t]{2}{*}{ E18 } & Ethyl hexadecanoate ${ }^{b}$ & 2179 & $29.33 \pm 0.06 \mathrm{~A}$ & $29.24 \pm 0.09 \mathrm{~A}$ & $29.29 \pm 0.02 \mathrm{~A}$ & $29.35 \pm 0.10 \mathrm{~A}$ & $29.16 \pm 0.02 \mathrm{~A}$ \\
\hline & $\begin{array}{l}\text { Total ethyl esters }(\mathrm{mg} / \mathrm{L}) \\
\text { Other esters }\end{array}$ & & $6.49 \pm 0.27 \mathrm{~A}$ & $120.76 \pm 3.29 \mathrm{C}$ & $72.23 \pm 3.38 \mathrm{~B}$ & $67.02 \pm 5.30 \mathrm{~B}$ & $65.40 \pm 2.28 \mathrm{~B}$ \\
\hline E19 & Isopentyl isopentanoate ${ }^{b}$ & 1290 & $3.67 \pm 0.01$ & $\operatorname{tr}$ & $\operatorname{tr}$ & $\operatorname{tr}$ & $\operatorname{tr}$ \\
\hline E20 & Methyl octanoate ${ }^{\text {a }}$ & 1384.1 & $1.57 \pm 0.13 \mathrm{C}$ & $0.93 \pm 0.08 \mathrm{~B}$ & $1.05 \pm 0.03 \mathrm{~B}$ & $0.71 \pm 0.01 \mathrm{AB}$ & $0.43 \pm 0.15 \mathrm{~A}$ \\
\hline E21 & Isoamyl hexanoate a & 1454.4 & $3.98 \pm 0.06 \mathrm{~B}$ & $3.89 \pm 0.03 \mathrm{~B}$ & $3.91 \pm 0.05 \mathrm{~B}$ & $3.54 \pm 0.02 \mathrm{~A}$ & $3.53 \pm 0.08 \mathrm{~A}$ \\
\hline E22 & Isoamyl lactate ${ }^{a}$ & 1562.8 & $194.55 \pm 4.00 \mathrm{~A}$ & $653.64 \pm 99.40 \mathrm{C}$ & $422.7 \pm 10.10 \mathrm{~B}$ & $400.48 \pm 5.28 \mathrm{~B}$ & $378.39 \pm 21.03 \mathrm{~B}$ \\
\hline E23 & Isoamyl octanoate ${ }^{\mathrm{b}}$ & 1650.8 & $111.54 \pm 40.15 \mathrm{AB}$ & $136.50 \pm 20.67 \mathrm{~B}$ & $127.35 \pm 2.89 \mathrm{AB}$ & $93.77 \pm 5.37 \mathrm{AB}$ & $43.26 \pm 14.53 \mathrm{~A}$ \\
\hline \multirow[t]{3}{*}{ E24 } & Methyl salicylate a & 1769.4 & $21.19 \pm 1.21 \mathrm{~A}$ & $20.03 \pm 0.94 \mathrm{~A}$ & $36.88 \pm 2.18 \mathrm{~B}$ & $31.75 \pm 1.20 \mathrm{~B}$ & $34.92 \pm 0.72 \mathrm{~B}$ \\
\hline & Total other esters & & $336.49 \pm 45.19 \mathrm{~A}$ & $814.99 \pm 120.97 \mathrm{C}$ & $\begin{array}{c}591.90 \pm 15.25 \\
\text { BC }\end{array}$ & $530.25 \pm 1.07 \mathrm{AB}$ & $460.53 \pm 5.71 \mathrm{AB}$ \\
\hline & $\begin{array}{l}\text { Total esters }(\mathrm{mg} / \mathrm{L}) \\
\text { Higher alcohols }\end{array}$ & & $48.95 \pm 0.16 \mathrm{~A}$ & $155.58 \pm 3.59 \mathrm{D}$ & $112.63 \pm 4.64 \mathrm{C}$ & $99.66 \pm 6.18 \mathrm{BC}$ & $97.16 \pm 1.02 \mathrm{~B}$ \\
\hline $\mathrm{H} 1$ & Isobutanol ${ }^{\mathrm{b}}(\mathrm{mg} / \mathrm{L})$ & 1089.1 & $19.56 \pm 0.49 \mathrm{~B}$ & $18.97 \pm 0.49 \mathrm{AB}$ & $19.67 \pm 0.74 \mathrm{~B}$ & $16.87 \pm 0.69 \mathrm{~A}$ & $18.48 \pm 0.62 \mathrm{AB}$ \\
\hline $\mathrm{H} 2$ & Isoamyl alcohol a (mg/L) & 1200.1 & $274.86 \pm 3.39 \mathrm{~A}$ & $265.07 \pm 8.42 \mathrm{~A}$ & $279.37 \pm 12.88 \mathrm{~A}$ & $254.94 \pm 11.52 \mathrm{~A}$ & $262.15 \pm 3.87 \mathrm{~A}$ \\
\hline $\mathrm{H} 3$ & 3-Methylpentanol a & 1319 & $127.34 \pm 1.68 \mathrm{~A}$ & $122.6 \pm 10.14 \mathrm{~A}$ & $129.33 \pm 4.90 \mathrm{~A}$ & $116.51 \pm 4.79 \mathrm{~A}$ & $118.42 \pm 0.30 \mathrm{~A}$ \\
\hline $\mathrm{H} 4$ & 1-Hexanol a $(\mathrm{mg} / \mathrm{L})$ & 1345.1 & $5.29 \pm 0.04 \mathrm{~B}$ & $5.20 \pm 0.19 \mathrm{~B}$ & $5.31 \pm 0.29 \mathrm{~B}$ & $4.04 \pm 0.10 \mathrm{~A}$ & $4.22 \pm 0.05 \mathrm{~A}$ \\
\hline
\end{tabular}


Table 3. Cont.

\begin{tabular}{|c|c|c|c|c|c|c|c|}
\hline Code & & $\mathrm{RI}^{1,2,3}$ & $\begin{array}{l}\text { Before Malolactic } \\
\text { Fermentation }\end{array}$ & $\begin{array}{l}\text { L. plantarum } \\
\text { C8-1 }\end{array}$ & $\begin{array}{l}\text { L. plantarum } \\
\text { Lp39 }\end{array}$ & $\begin{array}{l}\text { O. oeni } \\
\text { Oenos }\end{array}$ & $\begin{array}{l}\text { O. oeni } \\
\text { CiNe }\end{array}$ \\
\hline H5 & (E)-3-Hexen-1-ol ${ }^{\mathrm{a}}$ & 1356.2 & $6.00 \pm 3.87 \mathrm{~A}$ & $18.63 \pm 2.85 \mathrm{C}$ & $17.25 \pm 1.04 \mathrm{BC}$ & $1.83 \pm 1.23 \mathrm{~A}$ & $8.54 \pm 1.72 \mathrm{AB}$ \\
\hline H6 & (Z)-3-Hexen-1-ol a & 1377.2 & $337.11 \pm 21.35 \mathrm{~A}$ & $401.45 \pm 12.98 \mathrm{~A}$ & $370.44 \pm 14.70 \mathrm{~A}$ & $352.69 \pm 19.78 \mathrm{~A}$ & $348.44 \pm 11.02 \mathrm{~A}$ \\
\hline $\mathrm{H} 7$ & 2-Ethyl-1-hexanol a & 1480.4 & $7.79 \pm 0.48 \mathrm{~A}$ & $11.75 \pm 0.39 \mathrm{~B}$ & $11.81 \pm 0.01 \mathrm{~B}$ & $10.87 \pm 0.23 \mathrm{~B}$ & $11.96 \pm 1.00 \mathrm{~B}$ \\
\hline $\mathrm{H} 8$ & 3-Ethyl-4-methylpentanol ${ }^{\mathrm{b}}$ & 1501.1 & $35.39 \pm 1.69 \mathrm{~A}$ & $37.96 \pm 0.77 \mathrm{~A}$ & $38.54 \pm 0.99 \mathrm{~A}$ & $37.08 \pm 0.54 \mathrm{~A}$ & $36.09 \pm 2.06 \mathrm{~A}$ \\
\hline H9 & 2-Nonanol ${ }^{\mathrm{a}}$ & 1509.9 & $1.81 \pm 0.10 \mathrm{~A}$ & $1.73 \pm 0.03 \mathrm{~A}$ & $3.10 \pm 0.12 \mathrm{~B}$ & $1.80 \pm 0.19 \mathrm{~A}$ & $1.51 \pm 0.14 \mathrm{~A}$ \\
\hline $\mathrm{H} 10$ & 2,3-Butanediol ${ }^{b}$ & 1531.8 & $387.95 \pm 14.47 \mathrm{~A}$ & $317.36 \pm 38.57 \mathrm{~A}$ & $542.79 \pm 155.35 \mathrm{~A}$ & $336.66 \pm 54.59 \mathrm{~A}$ & $322.23 \pm 59.76 \mathrm{~A}$ \\
\hline H11 & $1-$ Octanol $^{\mathrm{a}}$ & 1549.2 & $26.11 \pm 3.24 \mathrm{~A}$ & $30.08 \pm 0.88 \mathrm{AB}$ & $34.38 \pm 0.03 \mathrm{BC}$ & $36.90 \pm 1.61 \mathrm{BC}$ & $38.89 \pm 2.46 \mathrm{C}$ \\
\hline H12 & 1-Decanol ${ }^{\mathrm{b}}$ & 1746.9 & $3.12 \pm 0.12 \mathrm{~A}$ & $2.43 \pm 0.01 \mathrm{~A}$ & $2.48 \pm 0.32 \mathrm{~A}$ & $2.60 \pm 0.27 \mathrm{~A}$ & $2.99 \pm 0.99 \mathrm{~A}$ \\
\hline H12 & Benzyl alcohol $^{a}$ & 1856.6 & $169.47 \pm 35.69 \mathrm{~A}$ & $275.53 \pm 6.46 \mathrm{~A}$ & $244.54 \pm 2.78 \mathrm{~A}$ & $210.57 \pm 16.29 \mathrm{~A}$ & $314.76 \pm 155.88 \mathrm{~A}$ \\
\hline \multirow[t]{2}{*}{ H13 } & 2-Phenylethanol a (mg/L) & 1889.3 & $25.74 \pm 1.53 \mathrm{AB}$ & $26.19 \pm 0.89 \mathrm{AB}$ & $29.76 \pm 0.20 \mathrm{~B}$ & $25.56 \pm 2.22 \mathrm{AB}$ & $21.98 \pm 2.68 \mathrm{~A}$ \\
\hline & $\begin{array}{l}\text { Total higher alcohols (mg/L) } \\
\text { Aldehydes/Ketones }\end{array}$ & & $326.55 \pm 5.52 \mathrm{~A}$ & $316.65 \pm 9.99 \mathrm{~A}$ & $335.5 \pm 14.29 \mathrm{~A}$ & $302.52 \pm 13.20 \mathrm{~A}$ & $308.04 \pm 7.33 \mathrm{~A}$ \\
\hline AK1 & Acetaldehyde ${ }^{\mathrm{b}}(\mathrm{mg} / \mathrm{L})$ & 655 & $34.23 \pm 2.06 \mathrm{~A}$ & $30.94 \pm 1.41 \mathrm{~A}$ & $31.23 \pm 1.55 \mathrm{~A}$ & $28.33 \pm 1.68 \mathrm{~A}$ & $29.30 \pm 0.77 \mathrm{~A}$ \\
\hline AK2 & 2,6-Dimethyl-4-heptanone ${ }^{\mathrm{b}}$ & 1170.5 & $196.54 \pm 18.68 \mathrm{~A}$ & $150.26 \pm 48.15 \mathrm{~A}$ & $186.86 \pm 13.33 \mathrm{~A}$ & $178.15 \pm 7.74 \mathrm{~A}$ & $147.30 \pm 40.39 \mathrm{~A}$ \\
\hline AK3 & Nonanal $^{\mathrm{b}}$ & 1390.9 & $3.92 \pm 2.55 \mathrm{~A}$ & $4.46 \pm 2.64 \mathrm{~A}$ & $3.31 \pm 0.88 \mathrm{~A}$ & $2.44 \pm 0.23 \mathrm{~A}$ & $2.24 \pm 0.41 \mathrm{~A}$ \\
\hline AK4 & Decanal a & 1495 & $0.81 \pm 0.05 \mathrm{~A}$ & $0.97 \pm 0.03 \mathrm{~A}$ & $1.10 \pm 0.11 \mathrm{~A}$ & $0.97 \pm 0.36 \mathrm{~A}$ & $1.32 \pm 0.68 \mathrm{~A}$ \\
\hline AK5 & Benzaldehyde $^{a}$ & 1524.2 & $38.50 \pm 17.71 \mathrm{~A}$ & $27.86 \pm 1.33 \mathrm{~A}$ & $28.22 \pm 3.00 \mathrm{~A}$ & $34.96 \pm 1.20 \mathrm{~A}$ & $71.62 \pm 53.00 \mathrm{~A}$ \\
\hline \multirow[t]{2}{*}{ AK6 } & 3,4-Dimethylbenzaldehyde ${ }^{\text {b }}$ & 1806.6 & $482.89 \pm 5.85 \mathrm{~A}$ & $458.21 \pm 23.11 \mathrm{~A}$ & $\begin{array}{c}550.95 \pm 28.40 \\
\mathrm{AB}\end{array}$ & $748.42 \pm 113.34 \mathrm{~B}$ & $517.37 \pm 21.26 \mathrm{~A}$ \\
\hline & $\begin{array}{l}\text { Total aldehydes/ketones }(\mathrm{mg} / \mathrm{L}) \\
\text { Fatty acids }\end{array}$ & & $34.95 \pm 2.06 \mathrm{~A}$ & $31.59 \pm 1.38 \mathrm{~A}$ & $32.00 \pm 1.57 \mathrm{~A}$ & $29.29 \pm 1.56 \mathrm{~A}$ & $30.04 \pm 0.78 \mathrm{~A}$ \\
\hline F1 & Acetic acid ${ }^{b}(\mathrm{mg} / \mathrm{L})$ & 1443.3 & $1.87 \pm 0.15 \mathrm{~A}$ & $2.32 \pm 0.49 \mathrm{~A}$ & $2.51 \pm 0.02 \mathrm{~A}$ & $1.97 \pm 0.01 \mathrm{~A}$ & $2.05 \pm 1.05 \mathrm{~A}$ \\
\hline F2 & Isobutyric acid ${ }^{\mathrm{a}}(\mathrm{mg} / \mathrm{L})$ & 1556.8 & $1.74 \pm 0.05 \mathrm{~A}$ & $1.61 \pm 0.08 \mathrm{~A}$ & $1.64 \pm 0.04 \mathrm{~A}$ & $1.61 \pm 0.16 \mathrm{~A}$ & $1.55 \pm 0.11 \mathrm{~A}$ \\
\hline F3 & Butyric acid a $(\mathrm{mg} / \mathrm{L})$ & 1615.9 & $1.04 \pm 0.09 \mathrm{~A}$ & $0.99 \pm 0.06 \mathrm{~A}$ & $1.09 \pm 0.07 \mathrm{~A}$ & $0.97 \pm 0.09 \mathrm{~A}$ & $0.86 \pm 0.05 \mathrm{~A}$ \\
\hline F4 & Isovaleric acid ${ }^{b}(\mathrm{mg} / \mathrm{L})$ & 1656.3 & $1.02 \pm 0.02 \mathrm{~A}$ & $1.02 \pm 0.01 \mathrm{~A}$ & $1.03 \pm 0.01 \mathrm{~A}$ & $1.19 \pm 0.04 \mathrm{~B}$ & $1.15 \pm 0.02 \mathrm{~B}$ \\
\hline F5 & Hexanoic acid a $(\mathrm{mg} / \mathrm{L})$ & 1821 & $2.74 \pm 0.35 \mathrm{~A}$ & $2.40 \pm 0.05 \mathrm{~A}$ & $2.43 \pm 0.08 \mathrm{~A}$ & $2.70 \pm 0.21 \mathrm{~A}$ & $2.33 \pm 0.08 \mathrm{~A}$ \\
\hline F6 & Octanoic acid ${ }^{\mathrm{a}}(\mathrm{mg} / \mathrm{L})$ & 2007.7 & $1.84 \pm 0.30 \mathrm{~B}$ & $1.53 \pm 0.03 \mathrm{AB}$ & $1.50 \pm 0.05 \mathrm{AB}$ & $1.48 \pm 0.13 \mathrm{AB}$ & $1.15 \pm 0.08 \mathrm{~A}$ \\
\hline \multirow[t]{3}{*}{ F7 } & n-Decanoic acid ${ }^{a}$ & 2178.8 & $173.81 \pm 3.09 \mathrm{~B}$ & $168.69 \pm 0.27 \mathrm{AB}$ & $167.34 \pm 0.35 \mathrm{~A}$ & $167.23 \pm 0.01 \mathrm{~A}$ & $166.89 \pm 0.10 \mathrm{~A}$ \\
\hline & Total fatty acids (mg/L) & & $10.42 \pm 0.66 \mathrm{~A}$ & $10.03 \pm 0.72 \mathrm{~A}$ & $10.36 \pm 0.24 \mathrm{~A}$ & $10.07 \pm 0.62 \mathrm{~A}$ & $9.25 \pm 1.40 \mathrm{~A}$ \\
\hline & Terpene derivatives & & & & & & \\
\hline $\mathrm{T} 1$ & $\alpha$-Ionone ${ }^{a}$ & 1528.5 & $2.95 \pm 0.13 \mathrm{~A}$ & $2.15 \pm 0.53 \mathrm{~A}$ & $2.67 \pm 0.15 \mathrm{~A}$ & $2.38 \pm 0.12 \mathrm{~A}$ & $3.30 \pm 0.47 \mathrm{~A}$ \\
\hline $\mathrm{T} 2$ & Linalool a & 1537.7 & $7.05 \pm 0.27 \mathrm{~A}$ & $6.69 \pm 0.19 \mathrm{~A}$ & $6.54 \pm 0.24 \mathrm{~A}$ & $13.26 \pm 0.24 \mathrm{~B}$ & $19.47 \pm 0.25 C$ \\
\hline T3 & 4-Terpineol a & 1597.5 & $2.40 \pm 0.66 \mathrm{~A}$ & $2.45 \pm 0.70 \mathrm{~A}$ & $2.19 \pm 0.12 \mathrm{~A}$ & $2.07 \pm 0.20 \mathrm{~A}$ & $2.27 \pm 0.26 \mathrm{~A}$ \\
\hline $\mathrm{T} 4$ & Hotrienol $^{\mathrm{b}}$ & 1600.9 & $0.80 \pm 0.02 \mathrm{~A}$ & $0.78 \pm 0.15 \mathrm{~A}$ & $0.79 \pm 0.04 \mathrm{~A}$ & $0.70 \pm 0.00 \mathrm{~A}$ & $0.88 \pm 0.01 \mathrm{~A}$ \\
\hline T5 & $\alpha$-Terpineol a & 1689 & $1.08 \pm 0.16 \mathrm{~A}$ & $1.76 \pm 0.06 \mathrm{~A}$ & $1.79 \pm 0.25 \mathrm{~A}$ & $3.32 \pm 0.01 \mathrm{~B}$ & $3.95 \pm 0.45 \mathrm{~B}$ \\
\hline T6 & D-Citronellol a & 1750.7 & $13.23 \pm 0.02 \mathrm{~A}$ & $12.83 \pm 2.81 \mathrm{~A}$ & $10.99 \pm 0.35 \mathrm{~A}$ & $11.07 \pm 0.09 \mathrm{~A}$ & $10.68 \pm 0.28 \mathrm{~A}$ \\
\hline T7 & $\beta$-Damascenone ${ }^{a}$ & 1808.4 & $20.45 \pm 1.12 \mathrm{~A}$ & $20.25 \pm 0.3 \mathrm{~A}$ & $21.53 \pm 0.72 \mathrm{~A}$ & $19.63 \pm 0.54 \mathrm{~A}$ & $19.44 \pm 1.56 \mathrm{~A}$ \\
\hline $\mathrm{T} 8$ & $\beta$-Ionone ${ }^{a}$ & 1916 & $1.10 \pm 0.04 \mathrm{C}$ & $0.94 \pm 0.00 \mathrm{~B}$ & $0.95 \pm 0.01 \mathrm{~B}$ & $0.74 \pm 0.01 \mathrm{~A}$ & $\operatorname{tr}$ \\
\hline T9 & Cadalene $^{\mathrm{b}}$ & 2150.9 & $9.04 \pm 0.78 \mathrm{~B}$ & $3.71 \pm 0.96 \mathrm{~A}$ & $4.27 \pm 0.36 \mathrm{~A}$ & $6.07 \pm 0.00 \mathrm{~A}$ & $5.49 \pm 0.43 \mathrm{~A}$ \\
\hline
\end{tabular}


Table 3. Cont.

\begin{tabular}{|c|c|c|c|c|c|c|c|}
\hline Code & & $\mathrm{RI}^{1,2,3}$ & $\begin{array}{l}\text { Before Malolactic } \\
\text { Fermentation }\end{array}$ & $\begin{array}{l}\text { L. plantarum } \\
\text { C8-1 }\end{array}$ & $\begin{array}{l}\text { L. plantarum } \\
\text { Lp39 }\end{array}$ & $\begin{array}{l}\text { O. oeni } \\
\text { Oenos }\end{array}$ & $\begin{array}{l}\text { O. oeni } \\
\text { CiNe }\end{array}$ \\
\hline $\mathrm{T} 10$ & $\begin{array}{c}\mathrm{TDN}^{4, \mathrm{~b}} \\
\text { Total terpene derivatives } \\
\text { Others }\end{array}$ & 1737.9 & $\begin{array}{c}1.70 \pm 0.04 \mathrm{C} \\
59.80 \pm 1.59 \mathrm{AB}\end{array}$ & $\begin{array}{c}1.31 \pm 0.00 \mathrm{~A} \\
52.86 \pm 2.34 \mathrm{~A}\end{array}$ & $\begin{array}{l}1.39 \pm 0.02 \mathrm{AB} \\
53.10 \pm 1.95 \mathrm{~A}\end{array}$ & $\begin{array}{c}1.46 \pm 0.07 \mathrm{AB} \\
60.72 \pm 0.70 \mathrm{AB}\end{array}$ & $\begin{array}{c}1.51 \pm 0.04 \mathrm{~B} \\
66.99 \pm 3.75 \mathrm{~B}\end{array}$ \\
\hline $\mathrm{O} 1$ & Styrene ${ }^{a}$ & 1255.5 & $10.79 \pm 0.79 \mathrm{~B}$ & $3.72 \pm 0.06 \mathrm{~A}$ & $3.97 \pm 0.13 \mathrm{~A}$ & $2.86 \pm 0.04 \mathrm{~A}$ & $2.99 \pm 0.03 \mathrm{~A}$ \\
\hline $\mathrm{O} 2$ & Dihydro-2-methyl-3(2H)-Thiophenone & 1527.4 & $124.16 \pm 1.35 \mathrm{D}$ & $47.02 \pm 2.21 \mathrm{BC}$ & $60.81 \pm 6.08 \mathrm{C}$ & $32.87 \pm 0.05 \mathrm{AB}$ & $21.98 \pm 7.52 \mathrm{~A}$ \\
\hline $\mathrm{O} 3$ & Methionol ${ }^{b}$ & 1708 & $2.67 \pm 0.34 \mathrm{BC}$ & $2.18 \pm 0.27 \mathrm{ABC}$ & $3.24 \pm 0.36 \mathrm{C}$ & $2.00 \pm 0.20 \mathrm{AB}$ & $1.58 \pm 0.04 \mathrm{~A}$ \\
\hline $\mathrm{O} 4$ & Naphthalene $^{\text {a }}$ & 1736.5 & $1.39 \pm 0.03 \mathrm{~A}$ & $1.40 \pm 0.00 \mathrm{~A}$ & $1.44 \pm 0.07 \mathrm{~A}$ & $1.43 \pm 0.05 \mathrm{~A}$ & $1.39 \pm 0.06 \mathrm{~A}$ \\
\hline O5 & 2-Methylnaphthalene ${ }^{b}$ & 1872.1 & $55.41 \pm 0.50 \mathrm{~A}$ & $45.41 \pm 2.98 \mathrm{~A}$ & $57.39 \pm 11.84 \mathrm{~A}$ & $43.52 \pm 5.36 \mathrm{~A}$ & $43.07 \pm 6.00 \mathrm{~A}$ \\
\hline O6 & Phenol $^{\text {a }}$ & 1967.6 & $56.30 \pm 7.84 \mathrm{~A}$ & $66.79 \pm 4.62 \mathrm{~A}$ & $60.66 \pm 2.41 \mathrm{~A}$ & $61.43 \pm 5.76 \mathrm{~A}$ & $58.42 \pm 2.04 \mathrm{~A}$ \\
\hline O7 & 4-Ethylguaiacol a & 1990.4 & $37.84 \pm 4.09 \mathrm{~A}$ & $56.90 \pm 1.81 \mathrm{~B}$ & $36.14 \pm 1.54 \mathrm{~A}$ & $34.27 \pm 0.69 \mathrm{~A}$ & $30.35 \pm 1.19 \mathrm{~A}$ \\
\hline O8 & 4-Ethylphenol a & 2107 & $68.85 \pm 28.63 \mathrm{~A}$ & $129.45 \pm 5.35 \mathrm{~B}$ & $34.47 \pm 0.93 \mathrm{~A}$ & $31.03 \pm 1.70 \mathrm{~A}$ & $27.79 \pm 0.73 \mathrm{~A}$ \\
\hline O9 & 2,3-Dihydrobenzofuran $\mathrm{b}$ & 2274.1 & $20.07 \pm 4.48 \mathrm{~A}$ & $17.03 \pm 0.12 \mathrm{~A}$ & tr & tr & $\operatorname{tr}$ \\
\hline $\mathrm{O} 10$ & $\begin{array}{l}\text { 1-Methylnaphthalene }{ }^{b} \\
\text { Total others }\end{array}$ & 1841 & $\begin{array}{c}1.00 \pm 0.02 \mathrm{~A} \\
378.47 \pm 48.07 \mathrm{~B}\end{array}$ & $\begin{array}{c}1.00 \pm 0.01 \mathrm{~A} \\
370.90 \pm 12.23 \mathrm{~B}\end{array}$ & $\begin{array}{c}1.02 \pm 0.03 \mathrm{~A} \\
259.14 \pm 18.46 \mathrm{~A}\end{array}$ & $\begin{array}{c}1.00 \pm 0.00 \mathrm{~A} \\
210.40 \pm 13.75 \mathrm{~A}\end{array}$ & $\begin{array}{c}1.00 \pm 0.01 \mathrm{~A} \\
188.57 \pm 5.48 \mathrm{~A}\end{array}$ \\
\hline
\end{tabular}

${ }^{1} \mathrm{RI}$ indicates retention indices. ${ }^{2}$ Data are expressed as mean \pm standard deviation of duplicate tests. Different letters in the same row indicate significant difference at $p<0.05$. tr: trace amount. ${ }^{3}$ Compounds in bold have OAVs $>1 .{ }^{4}$ TDN, 1,1,6-Trimethyl-1,2-dihydronaphthalene. ${ }^{\text {a }}$ Quantification was achieved using the corresponding standard. ${ }^{\mathrm{b}}$ Quantitation was carried out using the standard that had the same carbon atoms or the similar functional structure. 


\subsubsection{Esters}

Esters are the fruity scented volatile compounds in grapes, which are mainly extracted from the maceration process and contribute the varietal aromatic feature to wine [29,53]. Alcoholic fermentation could also result in the formation of esters via the activity of esterase released from yeasts [54]. Esters can be divided into acetate esters, ethyl esters, and other esters according to their structural natures [45].

Acetate esters appeared to be the dominant esters in the Cabernet Gernischt wine before the malolactic fermentation. They represented over $90 \%$ of the total esters content (Table 3 ). It has been reported that ethyl acetate exhibited the pineapple, fruity, solvent, and balsamic scents [55]. Its flavor notes could be significantly incorporated to the overall aroma in the Cabernet Gernischt wine due to its high OAV (Table 4). Isoamyl acetate had a concentration below $1 \mathrm{mg} / \mathrm{L}$ before malolactic fermentation (Table 3), but it still significantly contributed its fruity note to the overall aroma in the wine due to its low odor threshold [45]. The malolactic fermentation resulted in a decrease in ethyl acetate and isoamyl acetate (Table 3). It should be pointed that the wines treated with the L. plantarum strains maintained a higher content of these acetate esters than those with O. oeni strains. Even though a concentration reduction in acetate esters occurred after the malolactic fermentation, they were still higher than their odor thresholds, thus significantly affecting the overall aroma of wine.

The Cabernet Gernischt wine contained total ethyl esters of $6.49 \mathrm{mg} / \mathrm{L}$ before the malolactic fermentation (Table 3). A dramatic content increase occurred after the malolactic fermentation by these lactic acid bacteria strains. The sample with C8-1 exhibited the greatest accumulation in ethyl esters $(120.76 \mathrm{mg} / \mathrm{L})$. Regarding the individual ethyl ester, ethyl lactate, a volatile with fruity and buttery notes [45], experienced a significant increase after the malolactic fermentation, and its level was found to be $116.95 \mathrm{mg} / \mathrm{L}$ in the sample with C8-1. By contrast, ethyl butanoate had suprathreshold concentration before the malolactic fermentation, which contributed to banana, pineapple, and strawberry scents [45] (Table 3). However, the malolactic fermentation via all these strains significantly reduced its concentration below the odor threshold. This indicated that its aromatic contribution was no longer effective then. The ethyl nonanoate and diethyl succinate were also affected by the malolactic fermentation in terms of their concentrations. However, these volatiles exhibited the limited aromatic contribution to the wine due to their low OAVs. Regarding different strains, the L. plantarum strains delayed the content decrease of ethyl butanoate, ethyl hexanoate, ethyl octanoate, and ethyl isopentyl succinate in the wine. Meanwhile, ethyl lactate and diethyl succinate appeared to be accumulated faster with the L. plantarum strains. It was worth noting that 2-hydroxyisovaleric acid ethyl ester was only quantified in the wine with C8-1 strain. 
Table 4. Odor activity value of critical volatile compounds in Cabernet Gernischt wine before and after malolactic fermentation using L. plantarum (C8-1 and Lp39) and O. oeni (Oenos and CiNe).

\begin{tabular}{|c|c|c|c|c|c|c|c|c|}
\hline Volatile Compounds ${ }^{1}$ & $\begin{array}{l}\text { Odor } \\
\text { Threshold } \\
(\mu \mathrm{g} / \mathrm{L})\end{array}$ & Descriptor $^{2}$ & Aroma Series & $\begin{array}{c}\text { Before } \\
\text { Malolactic } \\
\text { Fermentation }\end{array}$ & $\begin{array}{l}\text { L. plantarum } \\
\text { C8-1 }\end{array}$ & $\begin{array}{l}\text { L. plantarum } \\
\text { Lp39 }\end{array}$ & $\begin{array}{l}\text { O. oeni } \\
\text { Oenos }\end{array}$ & $\begin{array}{l}\text { O. oeni } \\
\text { CiNe }\end{array}$ \\
\hline Ethyl acetate & $12,300[55]$ & Pineapple, fruity, solvent, balsamic [55] & $1,4,5[55]$ & $3.36 \pm 0.01 \mathrm{~B}$ & $2.74 \pm 0.03 \mathrm{~A}$ & $3.21 \pm 0.10 \mathrm{~B}$ & $2.59 \pm 0.07 \mathrm{~A}$ & $2.53 \pm 0.10 \mathrm{~A}$ \\
\hline Isoamyl acetate & $160[45]$ & Banana [45] & 1 [45] & $5.01 \pm 0.02 \mathrm{C}$ & $2.12 \pm 0.24 \mathrm{~B}$ & $2.24 \pm 0.09 \mathrm{~B}$ & $1.80 \pm 0.07 \mathrm{AB}$ & $1.26 \pm 0.23 \mathrm{~A}$ \\
\hline Ethyl butanoate & $400[45]$ & Banana, pineapple, strawberry [45] & $1[45]$ & $1.62 \pm 0.07 \mathrm{C}$ & $0.76 \pm 0.02 \mathrm{AB}$ & $0.91 \pm 0.06 \mathrm{~B}$ & $0.61 \pm 0.10 \mathrm{~A}$ & $0.54 \pm 0.06 \mathrm{~A}$ \\
\hline Ethyl hexanoate & $80[55]$ & Strawberry, apple, banana [55] & $4[55]$ & $6.79 \pm 0.01 \mathrm{C}$ & $3.29 \pm 0.46 \mathrm{AB}$ & $4.29 \pm 0.21 \mathrm{~B}$ & $3.19 \pm 0.16 \mathrm{AB}$ & $2.61 \pm 0.75 \mathrm{~A}$ \\
\hline Ethyl heptanoate & $2[56]$ & Winelike, brandy, fruity [56] & $1,6[56]$ & $0.71 \pm 0.00 \mathrm{~B}$ & $0.52 \pm 0.10 \mathrm{AB}$ & $0.56 \pm 0.04 \mathrm{AB}$ & $0.59 \pm 0.03 \mathrm{AB}$ & $0.32 \pm 0.16 \mathrm{~A}$ \\
\hline Ethyl lactate & $154,636[45]$ & Fruity, buttery [45] & $1,7[45]$ & $0.03 \pm 0.00 \mathrm{~A}$ & $0.76 \pm 0.02 \mathrm{C}$ & $0.46 \pm 0.02 \mathrm{~B}$ & $0.43 \pm 0.03 \mathrm{~B}$ & $0.42 \pm 0.02 \mathrm{~B}$ \\
\hline Isoamyl hexanoate & $2.7[57]$ & Fruity, banana, apple, pineapple, green [55] & $1,3[58]$ & $1.47 \pm 0.02 \mathrm{~B}$ & $1.44 \pm 0.01 \mathrm{~B}$ & $1.45 \pm 0.02 \mathrm{~B}$ & $1.31 \pm 0.01 \mathrm{~A}$ & $1.31 \pm 0.03 \mathrm{~A}$ \\
\hline Methyl salicylate & $40[59]$ & wintergreen, mint [60] & $3[58]$ & $0.53 \pm 0.03 \mathrm{~A}$ & $0.50 \pm 0.02 \mathrm{~A}$ & $0.92 \pm 0.05 \mathrm{~B}$ & $0.79 \pm 0.03 \mathrm{~B}$ & $0.87 \pm 0.02 \mathrm{~B}$ \\
\hline Isoamyl alcohol & $60,000[55]$ & Solvent, sweet, alcohol, nail polish [55] & $4,6,7[55]$ & $4.58 \pm 0.06 \mathrm{~A}$ & $4.42 \pm 0.14 \mathrm{~A}$ & $4.66 \pm 0.21 \mathrm{~A}$ & $4.25 \pm 0.19 \mathrm{~A}$ & $4.37 \pm 0.06 \mathrm{~A}$ \\
\hline 1-Hexanol & $1100[45]$ & Herbaceous, grass, woody [45] & $3[45]$ & $4.81 \pm 0.04 \mathrm{~B}$ & $4.73 \pm 0.17 \mathrm{~B}$ & $4.83 \pm 0.26 \mathrm{~B}$ & $3.67 \pm 0.09 \mathrm{~A}$ & $3.84 \pm 0.04 \mathrm{~A}$ \\
\hline 2-Phenylethanol & $14,000[45]$ & Roses, honey [45] & $2[45]$ & $1.84 \pm 0.11 \mathrm{AB}$ & $1.87 \pm 0.06 \mathrm{AB}$ & $2.13 \pm 0.01 \mathrm{~B}$ & $1.83 \pm 0.16 \mathrm{AB}$ & $1.57 \pm 0.19 \mathrm{~A}$ \\
\hline Butyric acid & $173[61]$ & Cheese, fatty [62] & $7[62]$ & $6.00 \pm 0.51 \mathrm{~A}$ & $5.75 \pm 0.34 \mathrm{~A}$ & $6.28 \pm 0.43 \mathrm{~A}$ & $5.61 \pm 0.53 \mathrm{~A}$ & $4.99 \pm 0.28 \mathrm{~A}$ \\
\hline Hexanoic acid & $420[45]$ & Cheese, fatty [45] & $7[45]$ & $6.52 \pm 0.82 \mathrm{~A}$ & $5.70 \pm 0.13 \mathrm{~A}$ & $5.78 \pm 0.19 \mathrm{~A}$ & $6.42 \pm 0.49 \mathrm{~A}$ & $5.54 \pm 0.19 \mathrm{~A}$ \\
\hline Octanoic acid & $500[45]$ & Rancid, cheese, fatty acid [45] & $7[45]$ & $3.68 \pm 0.60 \mathrm{~B}$ & $3.06 \pm 0.07 \mathrm{AB}$ & $3.00 \pm 0.11 \mathrm{AB}$ & $2.96 \pm 0.27 \mathrm{AB}$ & $2.30 \pm 0.16 \mathrm{~A}$ \\
\hline$\alpha$-Ionone & $2.6[63]$ & Flowery, violet [64] & $2[58]$ & $1.13 \pm 0.05 \mathrm{~A}$ & $0.83 \pm 0.20 \mathrm{~A}$ & $1.03 \pm 0.06 \mathrm{~A}$ & $0.92 \pm 0.05 \mathrm{~A}$ & $1.27 \pm 0.18 \mathrm{~A}$ \\
\hline$\beta$-Damascenone & 4 [65] & Sweet, exotic flowers, stewed apple [66] & $1,2,4[66]$ & $5.11 \pm 0.28 \mathrm{~A}$ & $5.06 \pm 0.08 \mathrm{~A}$ & $5.38 \pm 0.18 \mathrm{~A}$ & $4.91 \pm 0.14 \mathrm{~A}$ & $4.86 \pm 0.39 \mathrm{~A}$ \\
\hline$\beta$-Ionone & $0.09[45]$ & Violets, rose [66] & $2[45]$ & $12.27 \pm 0.46 \mathrm{C}$ & $10.47 \pm 0.00 \mathrm{~B}$ & $10.55 \pm 0.10 \mathrm{~B}$ & $8.28 \pm 0.10 \mathrm{~A}$ & $\operatorname{tr}$ \\
\hline
\end{tabular}

${ }^{1}$ Data are expressed as mean \pm standard deviation of duplicate tests. Different letters in the same row indicate significant difference at $p<0.05$. tr: trace amount. ${ }^{2}$ Aroma series: $1=$ Fruity, $2=$ Floral, 3 = Herbaceous, $4=$ Caramel, $5=$ Balsamic, $6=$ Chemical, $7=$ Fatty. 
Among other ester volatiles, the wines fermented by L. plantarum strains showed more other esters than those with $O$. oeni strains. Isoamyl hexanoate significantly contributed its fruity, banana, apple, pineapple, and green scents (Table 3) [55], which was found to present a higher concentration in L. plantarum fermented wine samples. Both isoamyl lactate and methyl salicylate increased their concentrations two times after the malolactic fermentation.

It has been reported that both O. oeni and L. plantarum strains could release alcohol acyltransferase and reverse esterase [67]. These enzymes cleave alcohols to form esters in wine, and their activities were strain-dependent [35,67-71]. Esterase is another critical enzyme released by lactic acid bacteria to affect the metabolism of esters during wine malolactic fermentation [19]. Lactic acid produced by malolactic fermentation could be further esterified under the activity of esterase to yield lactate-related esters, such as ethyl lactate and isoamyl lactate $[69,71]$. This could explain the significant accumulation of lactate-related esters in the present study. In our previous work, we noticed that the malolactic fermentation by C8-1 exhibited the longest duration (14 days for C8-1, 10 days for Lp39, 4 days for Oenos, and 6 days for $\mathrm{CiNe}$ ), which potentially extended the esterification of lactic acid. This resulted in a greater accumulation of ethyl lactate and isoamyl lactate in the wine with the C8-1 strain (Table 3). In addition, esterase could also exhibit the capacity of hydrolyzing esters in wine during malolactic fermentation [19]. In the present study, isoamyl acetate, ethyl butanoate, ethyl hexanoate, and ethyl octanoate decreased their concentrations in the wine by all these strains (Table 3). Our results were consistent with the previously reported studies $[13,35]$. It should be noted that the L plantarum strains had higher concentrations of these esters than those with the O. oeni strains, indicating that the L. plantarum could delay the aromatic loss in wine after malolactic fermentation.

\subsubsection{Higher Alcohols}

Wine alcoholic fermentation plays a vital role in generating higher alcohols [72]. Higher alcohols have been reported to contribute the desirable complexity when their concentration was below $300 \mathrm{mg} / \mathrm{L}$; when exceeding $400 \mathrm{mg} / \mathrm{L}$, they negatively affect the aromatic quality of wine [29]. In the present study, the malolactic fermentation did not significantly alter the level of higher alcohols (Table 3). Isoamyl alcohol, 2-phenylethanol, 1-hexanol, and isobutanol were found to be the dominant higher alcohols in all these samples. Isoamyl alcohol was described as the solvent, sweet, alcohol, and nail polish notes [55], whereas 2-phenylethanol possessed rose and honey scents [45]. 1-Hexanol could provide the herbaceous, grass, and woody flavors [45]. The flavor scents of these higher alcohols contributed to the overall aroma due to their high OAVs (Table 4). It should be noted that although 3-methylpentanol, (Z)-3-hexen-1-ol, 2,3-butanediol, and benzyl alcohol also exhibited high concentrations in these wine samples, they played quite limited roles in the overall aroma due to their high odor thresholds. Among strains, two L. plantarum strains were found to benefit the accumulation of (E)-3-hexen-1-ol, 2-nonanol and 2,3-butanediol after the malolactic fermentation (Table 3). It has been reported that odorless glycosidic precursors in wine could be cleaved into aromatic aglycones, such as 1-hexanol and (E)-2-hexen-1-ol, with L. plantarum during malolactic fermentation [9]. We speculated that the accumulation of (E)-3-hexen-1-ol and 1-hexanol in our study mainly resulted from the hydrolysis of their precursors.

\subsubsection{Aldehydes/Ketones}

The oxidation of unsaturated fatty acids in wine could lead to the formation of aldehydes. During the biosynthesis of fatty acids, some activated fatty acids could be condensed to yield ketones [73]. In the present study, acetaldehyde was found to be the major aldehyde/ketone before and after the malolactic fermentation (Table 3). An increase of 2,6-dimethyl-4-heptanone and 3,4-dimethylbenzaldehyde occurred in the wine after the malolactic fermentation. The accumulation of 3,4-dimethylbenzaldehyde was significantly greater in wine with Oenos. It should be noted that these aldehydes/ketones could exhibit limited aromatic contribution due to their sub-threshold concentrations. 


\subsubsection{Fatty Acids}

Alcoholic fermentation plays a primary role in yielding fatty acids in wine [74]. Aliphatic acids are formed during the yeast fermentation, whereas the aldehyde oxidation could lead to the formation of branched-chain fatty acids [73]. $n$-Decanoic acid was found to be the dominant fatty acid in the wine before the malolactic fermentation in this study (Table 3), while the rest of the fatty acids exhibited a moderate content in the wine. Butyric acid, hexanoic acid, and octanoic acid were found to have concentrations higher than their odor thresholds, indicating that their featured aromas (cheese and fatty notes from butyric acid and hexanoic acid; rancid, cheese, and fatty acid scents from octanoic acid) were incorporated into the wine $[45,62]$. The malolactic fermentation did not significantly alter the level of fatty acids in all the wine samples. Only a significant content reduction of octanoic acid was found in the wine with CiNe. It has been reported that lipase released from lactic acid bacteria could metabolize lipids, which elevates the concentration of volatile fatty acids in wine [75]. For instance, octanoic acid has been reported to increase after malolactic fermentation $[42,76,77]$. However, some studies reported that malolactic fermentation could reduce octanoic acid in wine $[3,34,76]$. The wine fermented by $\mathrm{CiNe}$ strain had a decrease level of octanoic acid, which was similar as these reports. We speculate that different wine matrix could regulate the secretion of lipase from lactic acid bacteria, which further alter the metabolism of fatty acids in wine $[3,76]$.

\subsubsection{Terpene Derivatives}

Terpenes are important grape secondary metabolites with distinctive varietal aroma profiles. They are extracted into wine during fermentation [72]. In this study, the malolactic fermentation did not significantly alter the total terpene derivatives (Table 3). In terms of the individual terpene derivative, $\alpha$-ionone, $\beta$-damascenone, and $\beta$-ionone were found to be the key components contributing their flavors notes, because of their suprathreshold concentrations before malolactic fermentation. A significant decrease in $\beta$-ionone occurred after the malolactic fermentation. However, the wine with the L. plantarum strains exhibited higher content of $\beta$-ionone than those with O. oeni strains. Our result was as opposite to a published study where $\beta$-ionone was accumulated after malolactic fermentation by O. oeni or L. plantarum [3]. It has been accepted that oxidative stress in wine could reduce the level of terpenes $[78,79]$. Meanwhile, L. plantarum strains have been reported to enhance the accumulation of acetaldehydes during malolactic fermentation [43]. Therefore, we speculated acetaldehydes produced in samples with L. plantarum strains could effectively reduce the oxidative stress and thus delayed the oxidation of $\beta$-ionone. Additionally, O. oeni strains have been reported to release $\beta$-glucosidase, and this enzyme could hydrolyze the precursor of linalool and $\alpha$-terpineol to yield these two terpene derivatives in wine malolactic fermentation $[4,32,33,80-83]$. In the present study, a significant accumulation of linalool and $\alpha$-terpineol was observed in the samples with O. oeni strains (Table 3). On the contrary, the L. plantarum strains in the present study did not exhibit a significant release of linalool and $\alpha$-terpineol, indicating that both strains might lack $\beta$-glucosidase due to either their genes or the matrix stress during malolactic fermentation $[17,18]$. It should be noted that linalool and $\alpha$-terpineol exhibited their concentrations below odor thresholds, limiting their flavor contributions to the wine overall aroma.

\subsubsection{Others}

Dihydro-2-methyl-3(2H)-thiophenone appeared to be the dominant other volatile before the malolactic fermentation (Table 3). The malolactic fermentation reduced its level, and the wine fermented with $O$. oeni strains exhibited lower content compared to those with L. plantarum strains. A significant increase on 4-ethylphenol was found in the wine with C8-1. It has been reported that 4-hydroxycinnamic acid could be decarboxylated to 4-ethylphenol by lactic acid bacteria, and 4-ehtylphenol could provide wine with a phenolic off-flavor [84-86]. In the present study, the C8-1 strain fermented wine did not contain detectable 3-hydroxycinnamic acid (Table 1), which indicated that a thorough conversion from 
4-hydroxycinnamic acid to 4-ehtylphenol might take place in the wine due to the enzymes released by the C8-1 strain. 2,3-Dihydrobenzofuran was found to disappear in these wine samples after the malolactic fermentation (except C8-1). The flavor contribution from all these other volatiles was limited due to their low concentrations.

\subsection{Aroma Profile}

A total of 17 volatile compounds were found to significantly contribute their flavor notes to the overall aroma in the Cabernet Gernischt wine due to their high odor activity values (Table 4). The wine before the malolactic fermentation primarily featured fatty, floral, caramel, and fruity aromas. The malolactic fermentation resulted in a modification of most of the aromatic features except the chemical and fatty (Figure 1). A decrease in the fruity aroma was observed for both strains, but a greater decrease observed for O. oeni strains than the L. plantarum strains. In addition, the wine with O. oeni strains showed a significant reduction in the intensity of floral aroma, whereas the L. plantarum strains retained a similar floral intensity as the wine before the malolactic fermentation. It should also be noted that the malolactic fermentation by the O. oeni strains also caused a decrease in the intensity of the herbaceous and balsamic series. The C8-1 strain fermented wine exhibited a similar balsamic aromatic strength as the wine before the malolactic fermentation. These indicated that malolactic fermentation using the L. plantarum strains could better preserve the aromatic feature in wine than the O. oeni strains.

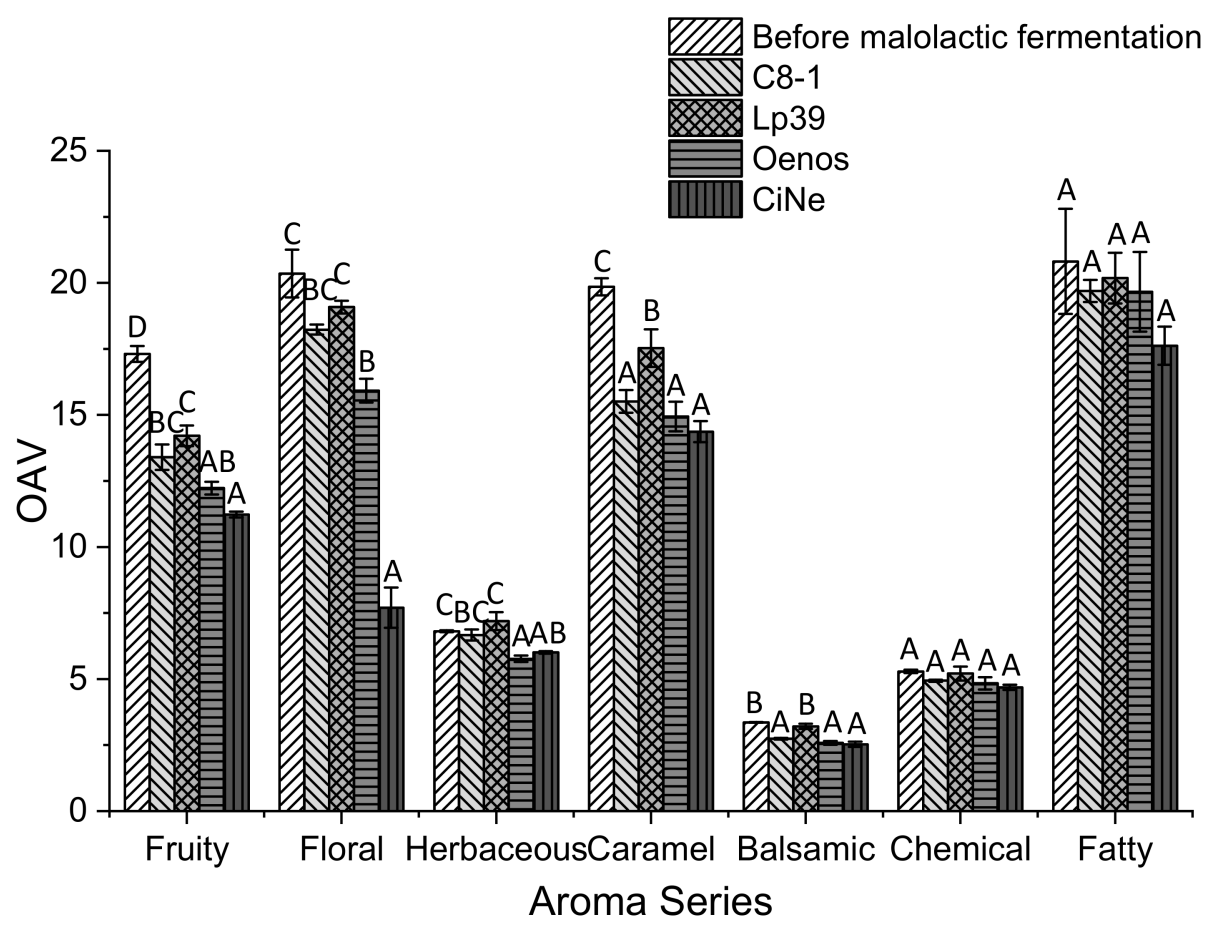

Figure 1. Aroma profile of Cabernet Gernischt wine before and after malolactic fermentation using L. plantarum (C8-1 and Lp39) and O. oeni (Oenos and CiNe). Aroma profile was calculated by summing up the odor activity values from Table 4 . Different letters within the same series indicate significant difference at $p<0.05$.

\subsection{Principal Component Analysis}

Principal component analysis was performed to characterize the overall quality of these wine samples by including phenolic compounds, biogenic amines, and volatile compounds as the variables (Figure 2a). The first and second components represented $50.94 \%$ and $26.44 \%$ of the total variance, respectively. More variables contributed to the positive F1 rather than negative F1. Most phenolic compounds appeared to correlate with the positive F1 and negative F2, while no specific distribution was found in biogenic amines. The Cabernet Gernischt wine before malolactic fermentation was 
positioned at the positive side of the F1, while the malolactic fermentation resulted in a negative shift in F1 for all strains. Strain-specific features were observed because the samples with L. plantarum strains moved to the positive axis of F2, while the samples with $O$. oeni assembled in its negative axis. Similar spatial distances were found between two L. plantarum strains and two O. oeni strains.
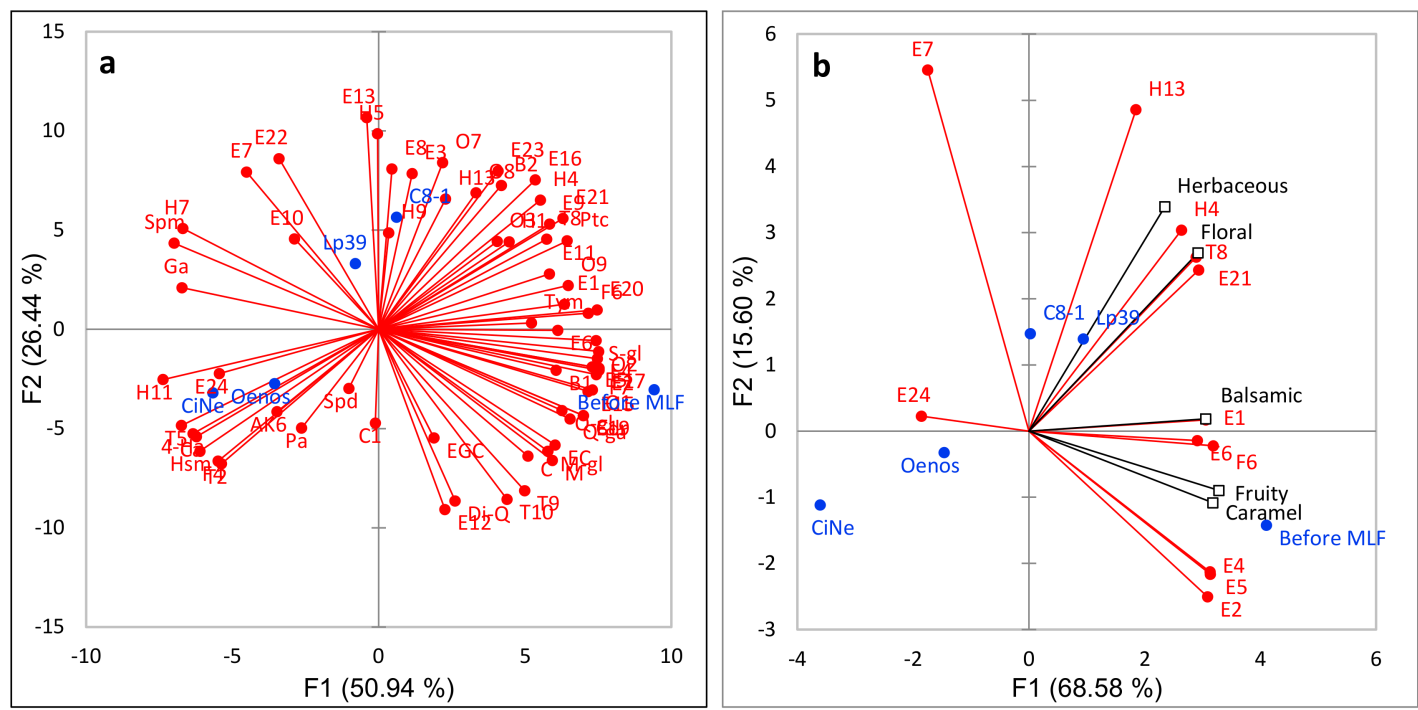

Figure 2. Principal component analysis of phenolic compounds, biogenic amines, and volatile compounds in Cabernet Gernischt wine before and after malolactic fermentation using L. plantarum (C8-1 and Lp39) and O. oeni (Oenos and CiNe) (a). Volatile compounds with significant odor activity value $(\mathrm{OAV})$ contributions are plotted in (b) using aroma series (black vectors) as supplementary variables. Only the variables (red vectors) with significant difference among samples (blue spots) are included in both biplots. Abbreviations: Before MLF, before malolactic fermentation; B1, Procyanin B1; B2, Procyanin B2; C1, Procyanin C1; C, Catechin; EC, Epicatechin; EGC, Epigallocatechin; M, Myricetin, M-gl, Myricetin-glucoside, Q-ga, Quercetin-galactoside; Q-gl, Quercetin-glucuronide; Di-Q, Dihydroquercetin, S-gl, Syringetin-glucoside; Ga, Gallic acid; Pa, Protocatechuic acid; Ca, Caffeic acid; 4-Ha, 4-Hydroxycinnamic acid; Hsm, Histamine; Spm, Spermine; Spd, Spermidine; Tym, Tyramine; Ptc, Putrescine; Cdr, Cadaverine. Codes of volatile compounds refer to Table 3.

Specific volatile compounds with significant contribution to aroma profile were separately analyzed in Figure $2 \mathrm{~b}$. The first two components represented $68.58 \%$ and $15.60 \%$ of the total variance, respectively. The strains and variables showed similar clustering and positioning as Figure 2a. Most volatiles pointed to the positive F1, while only two esters to the negative F1. The balsamic, fruity, and caramel series with ethyl acetate, ethyl heptanoate, octanoic acid, ethyl butanoate, ethyl hexanoate, isoamyl acetate, and methyl salicylate differentiated the samples before or after malolactic fermentation. The herbaceous and floral series with 2-phenylethanol, 1-hexanol, $\beta$-ionone, isoamyl hexanoate, and ethyl lactate better distinguished the strains' effects from L. plantarum and O. oeni.

\section{Conclusions}

In conclusion, the malolactic fermentation resulted in a decrease in the content of the total flavanols and total flavonols in wine, whereas an increase in the phenolic acids content was found in all the samples after malolactic fermentation. Cabernet Gernischt wine after malolactic fermentation with the L. plantarum strains exhibited a lower content of biogenic amines than those with the O. oeni strains. Malolactic fermentation using these strains also resulted in a significant increase in the total esters content. Modifications in the fruity, floral, herbaceous, caramel, and balsamic aroma series in Cabernet Gernischt wine was observed after malolactic fermentation. Compared to the O. oeni strains, wine fermented with the L. plantarum strains exhibited a better preservation of its aromatic features. Principal component analysis indicated that malolactic fermentation by different strains could alter the 
overall quality of wine towards specific directions. Some key volatile components either indicated the effects from malolactic fermentation or differentiated the specific strains.

Author Contributions: Conceptualization, B.-Q.Z. and B.-L.Z.; formal analysis, S.-Y.W., H.-Z.Z., Y.-B.L., R.-J.L., Y.-R.L.; data curation, S.-Y.W. and Y.-B.L.; writing, S.-Y.W. and B.-Q.Z. All authors have read and agreed to the published version of the manuscript.

Funding: This research was funded by Key Laboratory of Viticulture and Enology, Ministry of Agriculture (No. KLVE201701) and the Fundamental Research Funds for the Central Universities (2015ZCQ-SW-04).

Conflicts of Interest: The authors declare no conflict of interest.

\section{References}

1. Gil-Sánchez, I.; Bartolomé Suáldea, B.; Victoria Moreno-Arribas, M. Chapter 6-Malolactic Fermentation. In Red Wine Technology; Morata, A., Ed.; Academic Press: Cambridge, MA, USA, 2019; pp. 85-98. [CrossRef]

2. Pérez-Martín, F.; Izquierdo-Cañas, P.M.; Seseña, S.; García-Romero, E.; Palop, M.L. Aromatic compounds released from natural precursors by selected Oenococcus oeni strains during malolactic fermentation. Eur. Food Res. Technol. 2015, 240, 609-618. [CrossRef]

3. Pozo-Bayón, M.A.; G-Alegría, E.; Polo, M.C.; Tenorio, C.; Martín-Alvarez, P.J.; Calvo De La Banda, M.T.; Ruiz-Larrea, F.; Moreno-Arribas, M.V. Wine volatile and amino acid composition after malolactic fermentation: Effect of Oenococcus oeni and Lactobacillus plantarum starter cultures. J. Agric. Food Chem. 2005, 53, 8729-8735. [CrossRef] [PubMed]

4. Boido, E.; Lloret, A.; Medina, K.; Carrau, F.; Dellacassa, E. Effect of $\beta$-Glycosidase Activity of Oenococcus oeni on the Glycosylated Flavor Precursors of Tannat Wine during Malolactic Fermentation. J. Agric. Food Chem. 2002, 50, 2344-2349. [CrossRef] [PubMed]

5. Valdés La Hens, D.; Bravo-Ferrada, B.M.; Delfederico, L.; Caballero, A.C.; Semorile, L.C. Prevalence of Lactobacillus plantarum and Oenococcus oeni during spontaneous malolactic fermentation in Patagonian red wines revealed by polymerase chain reaction-denaturing gradient gel electrophoresis with two targeted genes. Aust. J. Grape Wine Res. 2015, 21, 49-56. [CrossRef]

6. Pramateftaki, P.V.; Metafa, M.; Kallithraka, S.; Lanaridis, P. Evolution of malolactic bacteria and biogenic amines during spontaneous malolactic fermentations in a Greek winery. Lett. Appl. Microbiol. 2006, 43, 155-160. [CrossRef] [PubMed]

7. López, I.; Núñez, R.L.; Lozano, C.T.; Larrea, F.R. Performance of malolactic fermentation by inoculation of selected Lactobacillus plantarum and Oenococcus oeni strains isolated from Rioja red wines. VITIS 2008, 47, $123-129$.

8. Restuccia, D.; Loizzo, M.R.; Spizzirri, U.G. Accumulation of Biogenic Amines in Wine: Role of Alcoholic and Malolactic Fermentation. Fermentation 2018, 4, 6. [CrossRef]

9. Iorizzo, M.; Testa, B.; Lombardi, S.J.; García-Ruiz, A.; Muñoz-González, C.; Bartolomé, B.; Moreno-Arribas, M.V. Selection and technological potential of Lactobacillus plantarum bacteria suitable for wine malolactic fermentation and grape aroma release. LWT 2016, 73, 557-566. [CrossRef]

10. Hernandez, T.; Estrella, I.; Perez-Gordo, M.; Alegria, E.G.; Tenorio, C.; Ruiz-Larrrea, F.; Moreno-Arribas, M.V. Contribution of malolactic fermentation by Oenococcus oeni and Lactobacillus plantarum to the changes in the nonanthocyanin polyphenolic composition of red wine. J. Agric. Food Chem. 2007, 55, 5260-5266. [CrossRef]

11. Lerm, E.; Engelbrecht, L.; Toit, M.D. Selection and characterisation of Oenococcus oeni and Lactobacillus plantarum South African wine isolates for use as malolactic fermentation starter cultures. S. Afr. J. Enol. Vitic. 2011, 32, 280-295. [CrossRef]

12. Capozzi, V.; Russo, P.; Ladero, V.; Fiocco, D.; Alvarez, M.A.; Grieco, F.; Spano, G. Biogenic Amines Degradation by Lactobacillus plantarum: Toward a Potential Application in Wine. Front. Microbiol. 2012, 3, 1-6. [CrossRef] [PubMed]

13. Brizuela, N.S.; Bravo-Ferrada, B.M.; Pozo-Bayón, M.Á.; Semorile, L.; Elizabeth Tymczyszyn, E. Changes in the volatile profile of Pinot noir wines caused by Patagonian Lactobacillus plantarum and Oenococcus oeni strains. Food Res. Int. 2018, 106, 22-28. [CrossRef] [PubMed] 
14. Brizuela, N.; Tymczyszyn, E.E.; Semorile, L.C.; La Hens, D.V.; Delfederico, L.; Hollmann, A.; Bravo-Ferrada, B. Lactobacillus plantarum as a malolactic starter culture in winemaking: A new (old) player? Electron. J. Biotechnol. 2018, 38, 10-18. [CrossRef]

15. Du Toit, M.; Engelbrecht, L.; Lerm, E.; Krieger-Weber, S. Lactobacillus: The Next Generation of Malolactic Fermentation Starter Cultures-An Overview. Food Bioprocess. Technol. 2011, 4, 876-906. [CrossRef]

16. Spano, G.; Beneduce, L.; Tarantino, D.; Zapparoli, G.; Massa, S. Characterization of Lactobacillus plantarum from wine must by PCR species-specific and RAPD-PCR. Lett. Appl. Microbiol. 2002, 35, 370-374. [CrossRef]

17. Grimaldi, A.; Bartowsky, E.; Jiranek, V. Screening of Lactobacillus spp. and Pediococcus spp. for glycosidase activities that are important in oenology. J. Appl. Microbiol. 2005, 99, 1061-1069. [CrossRef]

18. Grimaldi, A.; Bartowsky, E.; Jiranek, V. A survey of glycosidase activities of commercial wine strains of Oenococcus oeni. Int. J. Food Microbiol. 2005, 105, 233-244. [CrossRef]

19. Matthews, A.; Grimaldi, A.; Walker, M.; Bartowsky, E.; Grbin, P.; Jiranek, V. Lactic Acid Bacteria as a Potential Source of Enzymes for Use in Vinification. Appl. Environ. Microbiol. 2004, 70, 5715-5731. [CrossRef]

20. Liu, S.-Q. Malolactic fermentation in wine - beyond deacidification. J. Appl. Microbiol. 2002, 92, $589-601$. [CrossRef]

21. Li, S.; He, F.; Zhu, B.; Wang, J.; Duan, C. Comparison of phenolic and chromatic characteristics of dry red wines made from native Chinese grape species and Vitis vinifera. Int. J. Food Prop. 2016, 20, 2134-2146. [CrossRef]

22. He, F.; Liang, N.; Mu, L.; Pan, Q.; Wang, J.; Reeves, M.J.; Duan, C. Anthocyanins and their variation in red wines I. Monomeric anthocyanins and their color expression. Molecules 2012, 17, 1571-1601. [CrossRef] [PubMed]

23. Vivas, N.; Augustin, M.; Lonvaud-Funel, A. Influence of oak wood and grape tannins on the lactic acid bacterium CEenococcus oeni (Leuconostoc oenos, 8413). J. Sci. Food Agric. 2000, 80, 1675-1678. [CrossRef]

24. Campos, F.M.; Couto, J.A.; Hogg, T.A. Influence of phenolic acids on growth and inactivation of Oenococcus oeni and Lactobacillus hilgardii. J. Appl. Microbiol. 2003, 94, 167-174. [CrossRef] [PubMed]

25. Iorizzo, M.; Lombardi, S.J.; Renzo, T.D.; Testa, B.; Coppola, R.; Sorrentino, E. Effect of phenolic compounds on the growth and L-malic acid metabolism of Oenococcus oeni. J. Biotechnol. 2012, 6, 1225-1231. [CrossRef]

26. García-Ruiz, A.; Bartolomé, B.; Martínez-Rodríguez, A.J.; Pueyo, E.; Martín-Álvarez, P.J.; Moreno-Arribas, M.V. Potential of phenolic compounds for controlling lactic acid bacteria growth in wine. Food Control 2008, 19, 835-841. [CrossRef]

27. Devi, A.; Anu-Appaiah, K.A. Diverse physiological and metabolic adaptations by Lactobacillus plantarum and Oenococcus oeni in response to the phenolic stress during wine fermentation. Food Chem. 2018, 268, 101-109. [CrossRef]

28. Siebert, T.E.; Smyth, H.E.; Capone, D.L.; Neuwöhner, C.; Pardon, K.H.; Skouroumounis, G.K.; Herderich, M.J.; Sefton, M.A.; Pollnitz, A.P. Stable isotope dilution analysis of wine fermentation products by HS-SPME-GC-MS. Anal. Bioanal. Chem. 2005, 381, 937-947. [CrossRef]

29. Swiegers, J.H.; Pretorius, I.S. Yeast Modulation of Wine Flavor. In Advances in Applied Microbiology; Sariaslani, S., Gadd, G.M., Eds.; Academic Press: Cambridge, MA, USA, 2005; Volume 57, pp. 131-175. [CrossRef]

30. López, R.; López-Alfaro, I.; Gutiérrez, A.R.; Tenorio, C.; Garijo, P.; González-Arenzana, L.; Santamaría, P. Malolactic fermentation of Tempranillo wine: Contribution of the lactic acid bacteria inoculation to sensory quality and chemical composition. Int. J. Food Sci. Technol. 2011, 46, 2373-2381. [CrossRef]

31. Bartowsky, E.J.; Borneman, A.R. Genomic variations of Oenococcus oeni strains and the potential to impact on malolactic fermentation and aroma compounds in wine. Appl. Microbiol. Biotechnol. 2011, 92, 441-447. [CrossRef]

32. Antalick, G.; Perello, M.-C.; De Revel, G. Characterization of Fruity Aroma Modifications in Red Wines during Malolactic Fermentation. J. Agric. Food Chem. 2012, 60, 12371-12383. [CrossRef]

33. Bravo-Ferrada, B.M.; Hollmann, A.; Brizuela, N.; La Hens, D.V.; Tymczyszyn, E.; Semorile, L. Growth and consumption of l-malic acid in wine-like medium by acclimated and non-acclimated cultures of Patagonian Oenococcus oeni strains. Folia Microbiol. 2016, 61, 365-373. [CrossRef] [PubMed]

34. Knoll, C.; Fritsch, S.; Schnell, S.; Grossmann, M.; Krieger-Weber, S.; Du Toit, M.; Rauhut, D. Impact of different malolactic fermentation inoculation scenarios on Riesling wine aroma. World J. Microbiol. Biotechnol. 2012, 28, 1143-1153. [CrossRef] [PubMed] 
35. Brizuela, N.S.; Bravo-Ferrada, B.M.; Curilén, Y.; Delfederico, L.; Caballero, A.; Semorile, L.; Pozo-Bayón, M.Á.; Tymczyszyn, E.E. Advantages of Using Blend Cultures of Native L. plantarum and O. oeni Strains to Induce Malolactic Fermentation of Patagonian Malbec Wine. Front. Microbiol. 2018, 9, 2109. [CrossRef] [PubMed]

36. Guo, Y.Y.; Yang, Y.P.; Peng, Q.; Han, Y. Biogenic amines in wine: A review. Int. J. Food Sci. Technol. 2015, 50, 1523-1532. [CrossRef]

37. Izquierdo Cañas, P.M.; Gómez Alonso, S.; Ruiz Pérez, P.; Seseña Prieto, S.; García Romero, E.; Palop Herreros, M.L. Biogenic Amine Production by Oenococcus oeni Isolates from Malolactic Fermentation of Tempranillo Wine. J. Food Prot. 2009, 72, 907-910. [CrossRef]

38. Guerrini, S.; Mangani, S.; Granchi, L.; Vincenzini, M. Biogenic Amine Production by Oenococcus oeni. Curr. Microbiol. 2002, 44, 374-378. [CrossRef]

39. Cinquanta, L.; De Stefano, G.; Formato, D.; Niro, S.; Panfili, G. Effect of pH on malolactic fermentation in southern Italian wines. Eur. Food Res. Technol. 2018, 244, 1261-1268. [CrossRef]

40. Landete, J.M.; Ferrer, S.; Pardo, I. Biogenic amine production by lactic acid bacteria, acetic bacteria and yeast isolated from wine. Food Control 2007, 18, 1569-1574. [CrossRef]

41. Landete, J.M.; Ferrer, S.; Pardo, I. Which lactic acid bacteria are responsible for histamine production in wine? J. Appl. Microbiol. 2005, 99, 580-586. [CrossRef]

42. Sun, S.Y.; Chen, Z.X.; Jin, C.W. Combined influence of lactic acid bacteria starter and final $\mathrm{pH}$ on the induction of malolactic fermentation and quality of cherry wines. LWT 2018, 89, 449-456. [CrossRef]

43. Wang, S.; Li, S.; Zhao, H.; Gu, P.; Chen, Y.; Zhang, B.; Zhu, B. Acetaldehyde released by Lactobacillus plantarum enhances accumulation of pyranoanthocyanins in wine during malolactic fermentation. Food Res. Int. 2018, 108, 254-263. [CrossRef] [PubMed]

44. Gómez-Alonso, S.; Hermosín-Gutiérrez, I.; García-Romero, E. Simultaneous HPLC Analysis of Biogenic Amines, Amino Acids, and Ammonium Ion as Aminoenone Derivatives in Wine and Beer Samples. J. Agric. Food Chem. 2007, 55, 608-613. [CrossRef] [PubMed]

45. Cai, J.; Zhu, B.; Wang, Y.; Lu, L.; Lan, Y.; Reeves, M.J.; Duan, C. Influence of pre-fermentation cold maceration treatment on aroma compounds of Cabernet Sauvignon wines fermented in different industrial scale fermenters. Food Chem. 2014, 154, 217-229. [CrossRef] [PubMed]

46. Nemanic, J.; Vanzo, A.; Vrhovsek, U.J.V.J.O.G.R. Effect of red wine maceration techniques on oligomeric and polymeric proanthocyanidins in wine, cv. Blaufränkisch. VITIS 2002, 41, 47-51.

47. Jiménez, N.; Curiel, J.A.; Reverón, I.; De Las Rivas, B.; Muñoz, R. Uncovering the Lactobacillus plantarum WCFS1 Gallate Decarboxylase Involved in Tannin Degradation. Appl. Environ. Microbiol. 2013, 79, 4253-4263. [CrossRef]

48. Reverón, I.; Jiménez, N.; Curiel, J.A.; Peñas, E.; López De Felipe, F.; De Las Rivas, B.; Muñoz, R. Differential Gene Expression by Lactobacillus plantarum WCFS1 in Response to Phenolic Compounds Reveals New Genes Involved in Tannin Degradation. Appl. Environ. Microbiol. 2017, 83, e03387-16. [CrossRef]

49. Silva, I.; Campos, F.M.; Hogg, T.; Couto, J.A. Factors influencing the production of volatile phenols by wine lactic acid bacteria. Int. J. Food Microbiol. 2011, 145, 471-475. [CrossRef]

50. Vaquero, I.; Marcobal, Á.; Muñoz, R. Tannase activity by lactic acid bacteria isolated from grape must and wine. Int. J. Food Microbiol. 2004, 96, 199-204. [CrossRef]

51. María, E.T.; José María, L.; Inés, R.; Laura, S.; Blanca, D.L.R.; Rosario, M.O. A Lactobacillus plantarum esterase active on a broad range of phenolic esters. Appl. Environ. Microbiol. 2015, 81, 3235-3242. [CrossRef]

52. Arena, M.E.; Manca De Nadra, M.C. Biogenic amine production by Lactobacillus. J. Appl. Microbiol. 2001, 90, 158-162. [CrossRef]

53. Saerens, S.M.; Delvaux, F.; Verstrepen, K.J.; Van Dijck, P.; Thevelein, J.M.; Delvaux, F.R. Parameters affecting ethyl ester production by Saccharomyces cerevisiae during fermentation. Appl. Environ. Microbiol. 2008, 74, 454-461. [CrossRef] [PubMed]

54. Sumby, K.M.; Grbin, P.R.; Jiranek, V. Microbial modulation of aromatic esters in wine: Current knowledge and future prospects. Food Chem. 2010, 121, 1-16. [CrossRef]

55. Wang, S.; Li, Y.; Li, T.; Yang, H.; Ren, J.; Zhang, B.; Zhu, B. Dibasic Ammonium Phosphate Application Enhances Aromatic Compound Concentration in Bog Bilberry Syrup Wine. Molecules 2017, 22, 52. [CrossRef] [PubMed] 
56. Wu, Y.; Duan, S.; Zhao, L.; Gao, Z.; Luo, M.; Song, S.; Xu, W.; Zhang, C.; Ma, C.; Wang, S. Aroma characterization based on aromatic series analysis in table grapes. Sci Rep. 2016, 6, 31116. [CrossRef] [PubMed]

57. Narain, N.; Nigam, N.; De sousa galvão, M. CHAPTER 20-Passion Fruit. In Handbook of Fruit and Vegetable Flavors; Hui, Y.H., Ed.; John Wiley \& Sons, Inc.: Hoboken, NJ, USA, 2010; pp. 345-389. [CrossRef]

58. Noble, A.C.; Arnold, R.A.; Buechsenstein, J.; Leach, E.J.; Schmidt, J.O.; Stern, P.M. Modification of a Standardized System of Wine Aroma Terminology. Am. J. Enol. Vitic. 1991, 38, 143-146.

59. Leffingwell \& Associates. Odor \& Flavor Detection Thresholds in Water (In Parts per Billion). Available online: http://www.leffingwell.com/odorthre.htm (accessed on 21 September 2019).

60. The Good Scents Company Information System. Available online: http://www.thegoodscentscompany.com (accessed on 21 September 2019).

61. Ferreira, V.; López, R.; Cacho, J.F. Quantitative determination of the odorants of young red wines from different grape varieties. J. Sci. Food Agric. 2000, 80, 1659-1667. [CrossRef]

62. Sonni, F.; Moore, E.G.; Chinnici, F.; Riponi, C.; Smyth, H.E. Characterisation of Australian Verdelho wines from the Queensland Granite Belt region. Food Chem. 2016, 196, 1163-1171. [CrossRef]

63. Juan, F.S.; Cacho, J.; Ferreira, V.; Escudero, A. Aroma Chemical Composition of Red Wines from Different Price Categories and Its Relationship to Quality. J. Agric. Food Chem. 2012, 60, 5045-5056. [CrossRef]

64. Christensen, L.P.; Edelenbos, M.; Kreutzmann, S. Fruits and Vegetables of Moderate Climate. In Flavours and Fragrances: Chemistry, Bioprocessing and Sustainability; Berger, R.G., Ed.; Springer: Berlin/Heidelberg, Germany, 2007; pp. 135-187.

65. Sabon, I.; De Revel, G.; Kotseridis, Y.; Bertrand, A. Determination of Volatile Compounds in Grenache Wines in Relation with Different Terroirs in the Rhone Valley. J. Agric. Food Chem. 2002, 50, 6341-6345. [CrossRef]

66. Sánchez-Palomo, E.; Gómez García-Carpintero, E.; Alonso-Villegas, R.; González-Viñas, M.A. Characterization of aroma compounds of Verdejo white wines from the La Mancha region by odour activity values. Flavour Fragr. J. 2010, 25, 456-462. [CrossRef]

67. Costello, P.J.; Siebert, T.E.; Solomon, M.R.; Bartowsky, E.J. Synthesis of fruity ethyl esters by acyl coenzyme A: Alcohol acyltransferase and reverse esterase activities in Oenococcus oeni and Lactobacillus plantarum. J. Appl. Microbiol. 2013, 114, 797-806. [CrossRef] [PubMed]

68. Gammacurta, M.; Lytra, G.; Marchal, A.; Marchand, S.; Christophe Barbe, J.; Moine, V.; De Revel, G. Influence of lactic acid bacteria strains on ester concentrations in red wines: Specific impact on branched hydroxylated compounds. Food Chem. 2018, 239, 252-259. [CrossRef] [PubMed]

69. Delaquis, P.; Cliff, M.; King, M.; Girard, B.; Hall, J.; Reynolds, A. Effect of Two Commercial Malolactic Cultures on the Chemical and Sensory Properties of Chancellor Wines Vinified with Different Yeasts and Fermentation Temperatures. Am. J. Enol. Vitic. 2000, 51, 42-48.

70. Maicas, S.; Gil, J.-V.; Pardo, I.; Ferrer, S. Improvement of volatile composition of wines by controlled addition of malolactic bacteria. Food Res. Int. 1999, 32, 491-496. [CrossRef]

71. Gámbaro, A.; Boido, E.; Zlotejablko, A.; Medina, K.; Lloret, A.; Dellacassa, E.; Carrau, F. Effect of malolactic fermentation on the aroma properties of Tannat wine. Aust. J. Grape Wine Res. 2001, 7, 27-32. [CrossRef]

72. Ugliano, M.; Henschke, P.A. Yeasts and Wine Flavour. In Wine Chemistry and Biochemistry; Moreno-Arribas, M.V., Polo, M.C., Eds.; Springer: New York, NY, USA, 2009; pp. 313-392. [CrossRef]

73. Bakker, J.; Clarke, R.J. Wine Flavour Chemistry, 2nd ed.; Wiley-Blackwell: Hoboken, NJ, USA, 2011. [CrossRef]

74. Miranda-López, R.; Libbey, L.M.; Watson, B.T.; Mcdaniel, M.R. Identification of Additional Odor-Active Compounds in Pinot noir Wines. Am. J. Enol. Vitic. 1992, 43, 90-92.

75. Ugliano, M.; Moio, L. Changes in the Concentration of Yeast-Derived Volatile Compounds of Red Wine during Malolactic Fermentation with Four Commercial Starter Cultures of Oenococcus oeni. J. Agric. Food Chem. 2005, 53, 10134-10139. [CrossRef]

76. Knoll, C.; Fritsch, S.; Schnell, S.; Grossmann, M.; Rauhut, D.; Du Toit, M. Influence of pH and ethanol on malolactic fermentation and volatile aroma compound composition in white wines. LWT 2011, 44, 2077-2086. [CrossRef]

77. Sun, S.; Gong, H.; Liu, W.; Jin, C. Application and validation of autochthonous Lactobacillus plantarum starter cultures for controlled malolactic fermentation and its influence on the aromatic profile of cherry wines. Food Microbiol. 2016, 55, 16-24. [CrossRef]

78. Oliveira, J.M.; Oliveira, P.; Baumes, R.L.; Maia, O. Changes in aromatic characteristics of Loureiro and Alvarinho wines during maturation. J Food Compos. Anal. 2008, 21, 695-707. [CrossRef] 
79. Silva Ferreira, A.C.; Guedes De Pinho, P. Nor-isoprenoids profile during port wine ageing-Influence of some technological parameters. Anal. Chim. Acta 2004, 513, 169-176. [CrossRef]

80. Brizuela, N.S.; Bravo-Ferrada, B.M.; La Hens, D.V.; Hollmann, A.; Delfederico, L.; Caballero, A.; Tymczyszyn, E.E.; Semorile, L. Comparative vinification assays with selected Patagonian strains of Oenococcus oeni and Lactobacillus plantarum. LWT 2017, 77, 348-355. [CrossRef]

81. Mansfield, A.K.; Zoecklein, B.W.; Whiton, R.S. Quantification of Glycosidase Activity in Selected Strains of Brettanomyces bruxellensis and Oenococcus oeni. Am. J. Enol. Vitic. 2002, 53, 303-307.

82. Grimaldi, A.; Mclean, H.; Jiranek, V. Identification and Partial Characterization of Glycosidic Activities of Commercial Strains of the Lactic Acid Bacterium, Oenococcus oeni. Am. J. Enol. Vitic. 2000, 51, 362-369.

83. Ugliano, M.; Genovese, A.; Moio, L. Hydrolysis of Wine Aroma Precursors during Malolactic Fermentation with Four Commercial Starter Cultures of Oenococcus oeni. J. Agric. Food Chem. 2003, 51,5073-5078. [CrossRef]

84. Cavin, J.F.; Andioc, V.; Etievant, P.X.; Divies, C. Ability of Wine Lactic Acid Bacteria to Metabolize Phenol Carboxylic Acids. Am. J. Enol. Vitic. 1993, 44, 76-80.

85. Chatonnet, P.; Dubourdieu, D.; Boidron, J.N. The Influence of Brettanomyces/Dekkera sp. Yeasts and Lactic Acid Bacteria on the Ethylphenol Content of Red Wines. Am. J. Enol. Vitic. 1995, 46, 463-468.

86. Van Beek, S.; Priest, F.G. Decarboxylation of Substituted Cinnamic Acids by Lactic Acid Bacteria Isolated during Malt Whisky Fermentation. Appl. Environ. Microbiol. 2000, 66, 5322-5328. [CrossRef]

(C) 2020 by the authors. Licensee MDPI, Basel, Switzerland. This article is an open access article distributed under the terms and conditions of the Creative Commons Attribution (CC BY) license (http://creativecommons.org/licenses/by/4.0/). 